\title{
¿Sufren las mujeres inmigrantes una doble penalización salarial? Evidencia para España*
}

\author{
Hipólito Simón \\ Universidad de Alicante-IEI-IEB \\ Inés P. Murillo Huertas \\ Universidad de Extremadura
}

\begin{abstract}
Resumen
El artículo examina los salarios relativos de las mujeres inmigrantes en España mediante la Encuesta de Estructura Salarial a través de una doble comparación con los hombres inmigrantes y las mujeres nativas. El análisis empírico muestra que las mujeres inmigrantes presentan desventajas salariales significativas frente a ambos colectivos. Los resultados de los ejercicios de descomposición de estas diferencias salariales permiten constatar que mientras que una parte importante de la desventaja salarial frente a los varones inmigrantes no resulta explicada por las diferencias en dotaciones de características productivas, por el contrario, la brecha salarial frente a las mujeres nativas es explicada en buena medida por este elemento. En consecuencia, la evidencia obtenida para España no concuerda plenamente con la hipótesis de la existencia de una doble penalización salarial para las mujeres inmigrantes.

Palabras clave: discriminación salarial por razón de género, diferencias salariales nativosinmigrantes, datos emparejados empresa-trabajador, descomposición salarial de Juhn-MurphyPierce, descomposición de Fortin-Lemieux-Firpo.
\end{abstract}

Clasificación JEL: J15, J16, J31, J70.

\begin{abstract}
This study examines immigrant women relative wages in Spain as compared to those earned by immigrant men and native women on the basis of microdata from the Encuesta de Estructura Salarial. The results show that foreign-born women wages are significantly lower than those corresponding to both groups. It is also found that the wage gap compared to native women is explained largely by native women's better endowments of productive characteristics whereas, on the contrary, a significant part of the wage disadvantage relative to male immigrants is not explained by this element. As a consequence, empirical evidence for the case of Spain does not seem to fully support the hypothesis of a double wage penalty for immigrant women.
\end{abstract}

Keywords: gender wage discrimination, native-immigrant wage gap, matched employeremployee data, Juhn-Murphy-Pierce decomposition, Fortin-Lemieux-Firpo decomposition.

JEL classification: J15, J16, J31, J70.

* Este trabajo se ha beneficiado de la financiación del proyecto de investigación CSO2011-29943-C0302 del Ministerio de Educación, Cultura y Deporte. 


\section{Introducción}

La literatura económica ha documentado ampliamente la presencia de notables diferencias entre los salarios percibidos por nativos e inmigrantes y por hombres y mujeres (véanse Constant y Zimmerman, 2013 y Altonji y Blank, 1999 como acreditadas revisiones de estos ámbitos de la literatura). Por el contrario, son muy escasos los estudios que se han centrado en examinar el tratamiento salarial relativo que reciben las mujeres inmigrantes, aun a pesar de que se trata de una cuestión de notable interés, en tanto que las trabajadoras extranjeras podrían sufrir una doble penalización salarial al pertenecer de forma simultánea a dos colectivos, el de mujeres y el de inmigrantes, habitualmente penalizados en términos salariales (véanse, por ejemplo, Shamsuddin, 1998 y Hayfron, 2001).

Así, en los estudios relativos a la asimilación salarial de los inmigrantes en los países de destino, las principales aportaciones suelen analizar de forma exclusiva el colectivo de hombres (véanse, como ejemplos representativos, los trabajos seminales de Chiswick, 1978 y Carliner, 1980). Los análisis centrados en la evolución de los salarios relativos de las mujeres inmigrantes son, por el contrario, mucho más escasos $^{1}$. Una excepción notable en este sentido la constituye el estudio de Long (1980), donde se documenta que las pautas salariales de los inmigrantes difieren significativamente en función del sexo, tanto en el momento de la incorporación al país de destino como con el transcurso del tiempo. En el mismo sentido, estudios posteriores confirman las diferencias por género en cuanto a la magnitud y a los determinantes de los ingresos laborales de los inmigrantes en comparación con los obtenidos por los nativos (Adsera y Chiswick, 2007). Así, en línea con la hipótesis de que las mujeres inmigrantes planifican sus decisiones laborales como segundas perceptoras de renta y desde una perspectiva supeditada a los intereses familiares (Baker y Benjamin, 1997; Mincer, 1978), el estado civil y la situación familiar se configuran como variables determinantes de su tasa de participación y sus ingresos relativos (MacPherson y Stewart 1989; LeClere y McLaughlin, 1997; Duleep y Sanders, 1993). Más recientemente, la evidencia empírica señala como otros elementos relevantes en este sentido las características de los países de origen y de destino, la destreza con el nuevo idioma y la dotación de capital humano (Antecol et al., 2003, y Dustmann y Schmidt, 2000). Por último, cabe destacar el hallazgo de la existencia de una doble discriminación salarial para las mujeres inmigrantes más cualificadas, cuyos efectos tienden a mantenerse a lo largo de toda su carrera laboral (Beach y Worswick, 1993). Investigaciones posteriores en esta línea sugieren, además, que el efecto negativo asociado a ser mujer y extranjera podría ser aditivo, si bien las trabajadoras inmigrantes sufrirían una mayor discriminación salarial por motivos de género que por su procedencia (Shamsuddin, 1998, y Hayfron, 2001).

${ }^{1}$ Algo más numerosos son los trabajos que analizan las decisiones de las mujeres inmigrantes en términos de participación en el mercado laboral (REIMERS, 1985, o más recientemente, COBB-CLARK y CROSSEY, 2004). 
El objetivo de la presente investigación es examinar los salarios relativos de las trabajadoras inmigrantes en España, con el fin de contrastar si las mismas están penalizadas en términos salariales tanto por su condición de mujeres como por la de extranjeras. A pesar de que diversos estudios han indagado de forma específica en la asimilación laboral de las mujeres inmigrantes en España (Amuedo-Dorantes y De la Rica, 2007, 2011), hasta donde llega nuestro conocimiento ninguno de ellos ha examinado los ingresos salariales de dicho colectivo comparándolos de forma simultánea con los percibidos por los trabajadores varones extranjeros y por las trabajadoras nativas.

Con relación a lo anterior, cabe destacar que las mujeres inmigrantes en España muestran niveles acusados de segregación en función del tipo de empleos que ocupan tanto en comparación con los varones inmigrantes como en relación con las trabajadoras nativas (Del Río y Alonso-Villar, 2012). Esta circunstancia podría resultar de gran influencia de cara a la determinación de sus salarios, dado el efecto significativo que la segregación en empleos de bajos salarios ejerce en la práctica sobre los salarios relativos de los inmigrantes y de las mujeres. Así, por una parte, son diversos los estudios que han documentado cómo el colectivo de inmigrantes en España adolece de un grado de segregación laboral elevado y persistente en el tiempo (Alcobendas y Rodríguez-Planas, 2009; Alonso-Villar y del Río, 2013; Amuedo-Dorantes y De la Rica, 2011), así como que este elemento constituye uno de los principales factores explicativos de la desventaja salarial de los inmigrantes en comparación con los nativos (García-Pérez et al., 2012, Nicodemo y Ramos, 2012 y Simón et al., 2008a). De forma análoga, se ha constatado que la segregación de las trabajadoras españolas en ocupaciones y establecimientos de bajos salarios relativos es un importante elemento explicativo de la brecha salarial por razón de género, que opera principalmente a través de la obtención de unos menores complementos salariales (Amuedo-Dorantes y De la Rica, 2006; De la Rica et al., 2010; Simón et al., 2008b).

Con el objetivo de analizar los salarios relativos de las mujeres inmigrantes en España, el presente estudio utiliza las dos últimas olas de la Encuesta de Estructura Salarial, relativas a los años 2006 y 2010. Se trata de un periodo en el que los salarios relativos de las mujeres sufrieron en general un deterioro significativo en España a causa del impacto de la crisis económica (Murillo y Simón, 2014). En el análisis empírico se emplea una extensión de la metodología econométrica de descomposición propuesta por Juhn, Murphy y Pierce $(1991,1993)$ adaptada a los microdatos emparejados empresa-trabajador que ofrece la Encuesta de Estructura Salarial. La principal ventaja de esta técnica es que permite identificar en los resultados de la descomposición de las diferencias salariales el efecto aislado del establecimiento, haciendo posible cuantificar de este modo el impacto de la segregación por establecimiento. Asimismo, con el fin de extender el análisis a las diferencias salariales existentes en el conjunto de la distribución salarial, se utiliza también la metodología de descomposición propuesta por Fortin, Lemieux y Firpo (2011). Se trata de una técnica basada en la regresión cuantílica incondicionada y 
que permite cuantificar el impacto individual de cada variable explicativa sobre la brecha salarial a lo largo de la distribución de salarios.

El resto del texto se articula del siguiente modo. La sección segunda describe la base de datos utilizada, al tiempo que la sección tercera introduce las metodologías econométricas empleadas. La sección cuarta muestra y discute los principales hallazgos empíricos. Finalmente, la sección quinta subraya las principales conclusiones del estudio.

\section{Datos}

Esta investigación se desarrolla a partir de los microdatos de las olas de 2006 y 2010 de la Encuesta de Estructura Salarial (desde ahora, EES). Esta encuesta, elaborada por el Instituto Nacional de Estadística, constituye la muestra para España de la European Structure of Earnings Survey, una encuesta elaborada en los países miembros de la Unión Europea conforme a una metodología armonizada. Se trata de una encuesta representativa a nivel nacional realizada a empresas, que cubre a los empleados por cuenta ajena dados de alta en la Seguridad Social durante todo el mes de octubre del año de referencia en aquellos establecimientos de cualquier tamaño adscritos al Régimen general de la Seguridad Social cuya actividad económica se encuadre en las secciones B a S de la clasificación sectorial CNAE2009. En consecuencia, no cubre ciertos sectores de actividad como la agricultura o el servicio doméstico (y, hasta la ola de 2010, la rama de actividad correspondiente a la administración pública ${ }^{2}$. El diseño de la encuesta corresponde a un muestreo en dos etapas de asalariados a partir de las cuentas de cotización de sus empresas a la Seguridad Social, por lo que uno de sus rasgos más relevantes es la inclusión de microdatos emparejados empresa-trabajador (esto es, observaciones para varios asalariados en cada establecimiento).

La EES está formada por secciones cruzadas independientes que se elaboran con periodicidad cuatrienal, existiendo en la actualidad cuatro olas disponibles, correspondientes a los años 1995, 2002, 2006 y 2010. Con el transcurso del tiempo ha ido aumentando la información que contiene sobre las características de los trabajadores, ya que en la ola de 2002 se incorporaron por primera vez variables

${ }^{2}$ La ausencia de información en la encuesta sobre trabajadores no ocupados impide la aplicación de las técnicas estándar de corrección del sesgo de selección à la Heckman en las estimaciones econométricas (HECKMAN, 1979) y, en consecuencia, examinar la influencia de la selección en la participación laboral sobre la brecha salarial. Aun cuando esta circunstancia podría suponer una limitación significativa en el contexto del estudio desarrollado en esta investigación, de acuerdo a la información de la Encuesta de Población Activa las tasas de actividad de las mujeres inmigrantes son sistemáticamente superiores a la de las mujeres nativas (convergiendo incluso a lo largo del tiempo con las mayores tasas de actividad de los varones inmigrantes), por lo que el posible sesgo de selección en el caso de las mujeres inmigrantes supondría un inconveniente de menor magnitud que en el caso de las mujeres nativas. Con relación a esta cuestión, cabe subrayar también la ausencia en la EES de información relativa al estado civil y a la situación familiar de los trabajadores, variables que resultan fundamentales de cara al tratamiento del posible sesgo de selección y a la propia estimación de las ecuaciones salariales. 
informativas de la nacionalidad de los individuos y de la realización de tareas de supervisión. En el mismo sentido, también ha ido creciendo con el tiempo su cobertura, pues en la ola de 2002 se incluyeron por primera vez sectores de servicios no de mercado (educación, sanidad y otras actividades sociales); en la de 2006 los establecimientos con menos de 10 trabajadores y en la de 2010 el sector de actividad correspondiente a Administración Pública y defensa y Seguridad Social obligatoria. Debido a estas circunstancias, se ha optado por ceñir el análisis empírico que se desarrolla en la investigación a las olas de la EES de 2006 y 2010, al tratarse de las que presentan una mayor riqueza de información y, especialmente, una cobertura más completa del conjunto de la economía.

La encuesta proporciona información muy detallada sobre los salarios y las características de los trabajadores (nacionalidad, sexo, edad y educación); de sus puestos de trabajo (ocupación, antigüedad, tipo de contrato, tipo de jornada y realización de tareas de supervisión) y empresas (sector de actividad, tamaño, tipo de convenio colectivo y región). La información salarial incluye los distintos componentes que conforman el salario y abarca distintas referencias temporales (en concreto, incluye componentes salariales de carácter tanto mensual -salario base y complementos salariales de carácter mensual-como anual -salario total y complementos salariales anuales-). El concepto salarial utilizado en la investigación es el salario bruto por hora, calculado a partir del salario correspondiente a un mes de referencia representativo del conjunto del año en que se desarrolla la encuesta (octubre), dividido por el número de horas trabajadas en dicho mes. Los salarios están expresados en términos brutos y en su cálculo se incorpora cualquier tipo de pago por parte de las empresas, incluyendo comisiones, pluses por trabajo nocturnos y en fines de semana, así como el pago de horas extraordinarias.

Las variables explicativas que se han considerado en el análisis empírico abarcan tanto características de los individuos como de sus puestos de trabajo y empresas. En relación con las primeras, se trata de controles relativos al nivel de educación general (distinguiendo tres niveles: educación primaria, secundaria y terciaria) y la edad (diferenciando tres tramos: menos de 30 años, entre 30 y 45 años y más de 45 años). Las características de los puestos de trabajo son la ocupación (nueve categorías correspondientes a los grandes grupos ocupacionales); los años de antigüedad en el empleo actual y su forma cuadrática; el tipo de contrato (indefinido o de duración determinada); el tipo de jornada (tiempo completo o tiempo parcial) y la realización de tareas de supervisión. Finalmente, los atributos de las empresas son el sector (doce categorías) ${ }^{3}$; el tamaño (seis estratos); la región de ubicación y el tipo de convenio (distinguiendo entre convenio de empresa, de sector nacional y de sector infranacional).

\footnotetext{
${ }^{3}$ En relación con oleadas anteriores de la EES, en la ola de 2010 se emplean nuevas clasificaciones ocupacionales (CNO-11 en lugar de la CNO-94) y sectoriales (CNAE-2009 en lugar de CNAE-93). Con el fin de utilizar en el análisis empírico clasificaciones homogéneas en ambos casos, para las dos olas se han usado las secciones definidas según la CNAE-93 en el caso de los sectores (doce categorías) y los grandes grupos ocupacionales en el caso de las ocupaciones (nueve categorías).
} 
En otro orden de cosas, deben señalarse ciertas carencias de la EES que potencialmente podrían afectar a los resultados del análisis empírico. La primera es que, por sus características, se trata de una encuesta referida exclusivamente a trabajadores en situación legal, no abarcando, en consecuencia, a aquellos relacionados con la economía sumergida. La segunda es que no cubre ciertos segmentos del mercado de trabajo donde la presencia de inmigrantes es significativa, como el sector primario y el servicio doméstico. Como consecuencia de ello, la EES aparentemente tiende a subestimar, si bien de una forma no muy severa, el diferencial de salarios medios entre trabajadores nativos e inmigrantes (Simón et al., 2008a), una circunstancia que debe considerarse en la interpretación de los resultados. A pesar de todas las limitaciones señaladas, la EES presenta algunas ventajas sobre otras encuestas también empleadas en el análisis de las diferencias salariales por género y entre nativos e inmigrantes, como es el caso de la Muestra Continua de Vidas Laborales (MCVL) -véase, por ejemplo, Nicodemo y Ramos (2012)-. Así, además de ofrecer datos de carácter emparejado empresa-trabajador, la EES permite en la práctica calcular de forma muy fiable el salario por hora trabajada (variable dependiente más comúnmente empleada en los análisis sobre determinación salarial) al ofrecer una información completa y no censurada de los componentes salariales e información relativa al número de horas trabajadas ${ }^{4}$.

De cara al análisis empírico, se han filtrado aquellas observaciones con información no disponible sobre las principales variables de interés, así como las correspondientes a individuos mayores de 65 años o con salarios por hora inferiores a un euro o superiores a doscientos euros. Asimismo, los establecimientos con menos de dos observaciones han sido excluidos de la muestra, con el fin de permitir la correcta identificación de los efectos fijos por establecimiento en las estimaciones econométricas. Finalmente, con el fin de utilizar una cobertura sectorial homogénea, en la ola de 2010 se han eliminado las observaciones correspondientes a la sección O de la clasificación CNAE-2009 (Administración Pública y defensa; Seguridad Social obligatoria) $)^{5}$. Conforme es habitual en la literatura, en la investigación se considera inmigrantes a aquellos trabajadores con nacionalidad distinta de la española. Las muestras finales están conformadas por 3.417 mujeres inmigrantes, 6.876 hombres

\footnotetext{
${ }^{4}$ Sin embargo, la MCVL también presenta notables ventajas en su comparación con la EES, dado su mayor número de observaciones, su elevada fiabilidad al derivar de registros de carácter administrativo y, principalmente, su carácter longitudinal. Así, la MCVL ofrece información sobre toda la vida laboral del trabajador (con carácter retrospectivo para años anteriores a 2004) para un periodo temporal mucho más extenso que la EES, permitiendo controlar, en consecuencia, las posibles transiciones del empleo al desempleo de cada trabajador.

${ }^{5}$ Se ha comprobado que la no consideración de dicha sección de actividad no tiene efectos apreciables en las diferencias salariales entre hombres y mujeres inmigrantes, pero implica una ligera infravaloración de las diferencias salariales entre las mujeres nativas e inmigrantes (estos resultados están disponibles por parte de los autores ante su requerimiento). Esta circunstancia es coherente con el hecho de que la presencia de mujeres nativas en la sección de actividad $\mathrm{O}$ es significativamente mayor que la de hombres y mujeres inmigrantes, así como que en la misma se pagan en general salarios comparativamente mayores (pueden encontrarse más detalles de las diferencias salariales entre el sector público y el privado en España a partir de la EES en RAMOS et al., 2014).
} 
inmigrantes y 53.409 mujeres nativas para 2006 y por 4.272 mujeres inmigrantes, 6.584 hombres inmigrantes y 63.038 mujeres nativas para 2010 .

\section{Metodología}

En el artículo se utilizan dos metodologías para realizar descomposiciones de las diferencias salariales entre los colectivos comparados. La primera es una extensión de la metodología propuesta por Juhn, Murphy y Pierce $(1991,1993)$ adaptada a su uso con microdatos emparejados empresa-trabajador, la cual permite realizar una descomposición detallada del diferencial en el salario promedio entre dos colectivos, así como de su evolución en el tiempo. La segunda es la metodología elaborada por Fortin, Lemieux y Firpo (2011), que proporciona una descomposición detallada de las diferencias entre dos distribuciones salariales. A continuación se describen ambas técnicas.

\subsection{Descomposición de Juhn-Murpy-Pierce}

La extensión de la técnica de Juhn, Murphy y Pierce $(1991,1993)$ parte de la estimación separada para cada año de una ecuación salarial semilogarítmica minceriana con la forma:

$$
w_{i j}=X_{i} \beta+\varepsilon_{i j}+\alpha_{j}
$$

Donde $w_{i j}$ corresponde al logaritmo del salario bruto por hora del trabajador $i$ que presta sus servicios en el establecimiento $j ; X_{i}$ es un vector de variables explicativas individuales más un término constante; $\beta$ es un vector de parámetros a estimar; $\varepsilon_{i j}$ es un término de error aleatorio y $\alpha_{j}$ representa un término de error correspondiente al establecimiento $j$ e invariante para los individuos pertenecientes al mismo establecimiento.

En la línea de la recomendación de Oaxaca y Ransom (1994) y Neumark (1988), se ha empleado en la descomposición como estructura salarial de referencia la correspondiente al conjunto de individuos de la economía. Así, tras estimar la estructura salarial con la muestra correspondiente a todos los trabajadores y obtener los valores de $\hat{\beta}, \sigma$ y $\eta$, en función de las propiedades del estimador de mínimos cuadrados ordinarios el salario medio del colectivo $\mathrm{s}$ ( $\mathrm{s}=$ mujeres nativas, mujeres inmigrantes, hombres inmigrantes) se puede expresar como:

$$
\bar{w}^{s}=\bar{X}^{s} \hat{\beta}+\sigma \bar{\theta}^{s}+\eta \bar{\lambda}^{s} \text { donde } \bar{\theta} \sim(0,1), \bar{\lambda} \sim(0,1)
$$

Donde $\bar{w}^{s}$ es el salario medio del colectivo $s ; \bar{X}^{s}$ es un vector que contiene el valor medio muestral de las características observadas del colectivo $s ; \hat{\beta}$ es el vector de 
parámetros estimados conjuntamente para todos los individuos de la economía a partir de la ecuación [1]; $\bar{\theta}^{s}$ es el residuo salarial estandarizado promedio del colectivo $s$; $\sigma$ es la desviación estándar de los residuos salariales de la muestra conjunta; $\bar{\lambda}^{s}$ es el efecto fijo por establecimiento estandarizado promedio del colectivo $s$ y $\eta$ es la desviación estándar de los efectos fijos por establecimiento de la muestra conjunta.

Empleando la estructura salarial de referencia en la descomposición, la diferencia en el salario por hora medio de los dos colectivos a comparar (mujeres nativas e inmigrantes y hombres y mujeres inmigrantes, respectivamente), al que denominamos $D$, se puede expresar como:

$$
D=\bar{w}^{a}-\bar{w}^{b}=\left(\bar{X}^{a}-\bar{X}^{b}\right) \hat{\beta}+\left(\bar{\theta}^{a}-\bar{\theta}^{b}\right) \sigma+\left(\bar{\lambda}^{a}-\bar{\lambda}^{b}\right) \eta=\Delta \bar{X} \hat{\beta}+\Delta \bar{\theta} \sigma+\Delta \bar{\lambda} \eta
$$

Donde el operador $\Delta$ indica la diferencia entre los dos colectivos en el promedio de la variable a la que precede y los superíndices $a$ y $b$ corresponden, respectivamente, a cada uno de los dos colectivos comparados en cada caso.

La ecuación [3] permite cuantificar qué proporción de la brecha salarial entre los dos colectivos se explica, alternativamente, por diferencias en las características productivas observadas de cada uno de ellos; por la influencia de factores inobservables o por el establecimiento de pertenencia. Así, el primer término del lado derecho de la ecuación corresponde a la parte del diferencial salarial atribuible a las diferencias entre colectivos en las características observadas valoradas a los precios de mercado (coincidiendo el mismo con el componente «explicado» en la descomposición estándar de Oaxaca-Blinder). El segundo término captura el efecto de la diferencia en los residuos salariales estandarizados promedio de los colectivos, multiplicada por la dispersión de la distribución de los residuos (captando así la influencia en los salarios de los factores inobservables). Por último, el tercer componente aproxima la influencia del establecimiento, la cual viene determinada tanto por la intensidad de la segregación relativa de cada colectivo entre establecimientos como por la magnitud de la dispersión de las diferencias salariales entre establecimientos.

El cambio experimentado por la brecha salarial promedio entre dos colectivos entre los años 2006 y 2010 (denominados $A$ y $B$ en la ecuación), puede expresarse, a su vez, como:

$$
\begin{gathered}
D_{B}-D_{A}=\left(\Delta \bar{X}_{B}-\Delta \bar{X}_{A}\right) \hat{\beta}_{B}+\Delta \bar{X}_{A}\left(\hat{\beta}_{B}-\hat{\beta}_{A}\right)+\left(\Delta \bar{\theta}_{B}-\Delta \bar{\theta}_{A}\right) \sigma_{B}+ \\
+\Delta \bar{\theta}_{A}\left(\sigma_{B}-\sigma_{A}\right)+\left(\Delta \bar{\lambda}_{B}-\Delta \bar{\lambda}_{A}\right) \eta_{B}+\Delta \bar{\lambda}_{A}\left(\eta_{B}-\eta_{A}\right)
\end{gathered}
$$

De acuerdo con la ecuación [4], el cambio en la magnitud del diferencial salarial entre dos momentos del tiempo puede deberse a seis factores distintos, cada uno de ellos capturado por el correspondiente término de la descomposición. El primero es que se modifiquen las dotaciones relativas de características productivas observadas de los dos colectivos. El segundo es que la estructura general de diferenciales salariales de la economía experimente modificaciones. El tercero pasa por cambios en el efecto 
relativo de los factores inobservados que afecten a los residuos salariales promedio de los dos colectivos. El cuarto es que haya modificaciones en el grado de dispersión de los residuos salariales (y, por lo tanto, en la penalización asociada a la diferencia en los residuos estandarizados promedio). El quinto es que la diferencia en los efectos fijos por establecimiento promedio de los dos colectivos ( $\mathrm{y}$, en consecuencia, en la intensidad de la segregación por establecimiento) varíe con el tiempo. Por último, el sexto recoge la posibilidad de que se den modificaciones en la dispersión salarial entre establecimientos que impliquen, a su vez, cambios en la magnitud de la penalización salarial que sufre el colectivo segregado en establecimientos de bajos salarios relativos.

\subsection{Descomposición de Fortin-Lemieux-Firpo}

La técnica propuesta por Fortin, Lemieux y Firpo (2011), por su parte, permite desarrollar empíricamente descomposiciones de diferencias entre dos distribuciones de una variable. La misma se basa en el uso de la función de influencia recentrada y, en última instancia, proporciona una descomposición de las diferencias que se dan entre las distribuciones en el valor de estadísticos distribucionales como los cuantiles, en función de las diferencias que existen en las características observadas y en los rendimientos de las características, respectivamente. Se trata de un procedimiento que presenta una ventaja reseñable en relación con técnicas equiparables propuestas en la literatura económica que también permiten desarrollar empíricamente descomposiciones de diferencias entre distribuciones a partir de la construcción de distribuciones contrafactuales (DiNardo, Fortin y Lemieux, 1996; Juhn, Murphy y Pierce, 1993; Machado y Mata, 2005; Melly, 2005, 2006). Así, mientras que estas técnicas consisten en descomposiciones agregadas que, salvo excepciones parciales, proporcionan exclusivamente los efectos separados del conjunto de características y rendimientos, la metodología de Fortin, Lemieux y Firpo (2011) proporciona una descomposición detallada que permite conocer, además, la aportación individual de cada variable explicativa considerada en el análisis a través de los componentes de características y rendimientos.

Dicha técnica se basa en la estimación de una regresión donde la variable independiente (el salario) es sustituida por una transformación de la misma, la función de influencia recentrada (recentered influence function; desde ahora, RIF) para, con posterioridad, desarrollar una descomposición estándar a la Oaxaca-Blinder de cualquier estadístico distribucional basada en los resultados de la regresión.

La función de influencia mide el efecto en estadísticos distribucionales de pequeños cambios en la distribución subyacente. Así, para un estadístico distribucional dado de la distribución $F_{W}, v(F)$, esta función mide la importancia que tiene cada observación en la conformación del valor de dicho estadístico. Fortin, Lemieux y Firpo (2011) sugieren utilizar una versión recentrada de la función de influencia tras añadir el estadístico de interés, $\operatorname{RIF}(W)=v(F)+I F(W)$, ya que la misma tiene como valor 
esperado el propio estadístico $v(F)$ (en la medida en que la esperanza de la función de influencia con respecto a la distribución de $W$ es, por definición, cero). En el caso de los cuantiles $Q_{\theta}$ de la distribución marginal incondicionada $F_{W}$, la función de influencia, $\operatorname{IF}\left(W, Q_{\theta}\right)$, se define de la siguiente forma:

$$
I F\left(W / Q_{\theta}\right)=\frac{\theta-l\left\{W<Q_{\theta}\right\}}{f_{W}\left(Q_{\theta}\right)}
$$

Donde $l\{\cdot\}$ es una función indicador y $f_{W}$ es la función de densidad de la distribución marginal de $W$ evaluada en $Q_{\theta}$.

Dado que la función de influencia recentrada, $\operatorname{RIF}\left(W, Q_{\theta}\right)$, es igual a $Q_{\theta}+\operatorname{IF}(W$, $Q_{\theta}$ ), entonces se cumple que:

$$
\operatorname{RIF}\left(W / Q_{\theta}\right)=Q_{\theta}+\frac{\theta-l\left\{W<Q_{\theta}\right\}}{f_{W}\left(Q_{\theta}\right)}
$$

Así pues, la función RIF puede computarse empíricamente en el caso de los cuantiles mediante una inversión local, tras el cálculo de la variable dummy $l\left\{W<Q_{\theta}\right\}$ (la cual especifica si el valor de $W$ es mayor o menor que $Q_{\theta}$ ), la estimación del cuantil de la muestra $Q_{\theta}$ y la estimación mediante funciones de densidad kernel de la correspondiente función de densidad $f_{W}$ evaluada en $Q_{\theta}$.

Tras el cálculo de la función RIF para el cuantil, se dispone de un valor de la variable transformada para cada observación de la muestra. En la medida en que el efecto del cambio en la distribución de una variable explicativa en el cuantil puede expresarse, ceteris paribus, como el efecto parcial promedio de esa variable en la esperanza condicionada de su función RIF, y asumiendo que la esperanza condicionada de la función RIF puede ser modelizada como una función lineal de las variables explicativas, estos valores puede ser utilizados para la estimación mediante mínimos cuadrados ordinarios de una regresión de la variable RIF en el vector de variables explicativas. Los coeficientes estimados en la misma pueden ser interpretados como el efecto de un aumento en el valor promedio de una variable explicativa en el cuantil de la distribución (este tipo de estimación es denominada regresión cuantílica incondicionada; para más detalles, véase Firpo, Fortin y Lemieux, 2009).

Los coeficientes estimados de dicha regresión pueden emplearse para el cálculo en diferentes cuantiles de la distribución de una descomposición estándar a la Oaxaca-Blinder. En la práctica, en el desarrollo de la misma se ha empleado como estructura salarial de referencia en la descomposición la correspondiente al pool de los dos colectivos implicados en la comparación. Asimismo, para evitar el problema de identificación que surge en este tipo de descomposición, asociado al hecho de que la elección de una referencia específica en cada grupo de variables ficticias puede afectar en la práctica a los resultados de la descomposición detallada a través de la aportación relativa de cada variable explicativa al componente de rendimientos (Oaxaca y Ransom, 1999), en la estimación de la ecuación se ha adoptado la estrategia 
de normalización de variables ficticias sugerida por Yun (2005). Esto permite estimar más apropiadamente la contribución real de cada variable al componente de rendimientos de la descomposición ${ }^{6}$.

La descomposición toma, en consecuencia, la siguiente forma:

$$
\Delta Q_{\theta}=\left(\bar{X}^{a}-\bar{X}^{b}\right) \hat{\gamma}_{Q_{\theta}}^{*}+\left\{\bar{X}^{a}\left(\hat{\gamma}_{Q_{\theta}}^{b}-\hat{\gamma}_{Q_{\theta}}^{*}\right)+\bar{X}^{b}\left(\hat{\gamma}_{Q_{\theta}}^{*}-\hat{\gamma}_{Q_{\theta}}^{a}\right)\right\}
$$

Donde $\Delta_{Q_{\theta}}$ es la diferencia en el cuantil $Q_{\theta}$ de las distribuciones salariales de los dos colectivos a comparar; $\bar{X}^{a}$ y $\bar{X}^{b}$ son las características observadas promedio en cada uno de ellos y $\hat{\gamma}_{Q_{\theta}}^{a}, \hat{\gamma}_{Q_{\theta}}^{b}$ y $\hat{\gamma}_{Q_{\theta}}^{*}$ son los coeficientes estimados tras la regresión de la variable RIF del cuantil $Q_{\theta}$ sobre el conjunto de variables explicativas para cada colectivo y el pool de ambos, respectivamente. El primer componente del lado derecho de la ecuación representa el efecto sobre el diferencial entre distribuciones originado por diferencias en características (o componente «explicado»), mientras que el segundo corresponde al efecto de los coeficientes (o componente «no explicado»). Tal y como se ha señalado con anterioridad, en cada uno de ellos se puede distinguir la aportación diferenciada de cada factor explicativo individual.

\section{Resultados}

\subsection{Evidencia descriptiva}

Los Cuadros 1 y 2 y los Gráficos 1 y 2 contienen información sobre la brecha salarial entre hombres y mujeres inmigrantes y entre mujeres nativas e inmigrantes, respectivamente, medida en logaritmos del salario por hora (las correspondientes funciones de densidad de las distribuciones salariales de cada uno de los colectivos aparecen en los Gráficos A.1 y A.2 del anexo). A partir de esta información se constata que existe una significativa desventaja salarial de las mujeres inmigrantes tanto frente a los hombres inmigrantes $(0,132$ puntos logarítmicos en 2006 y 0,124 en 2010) como frente a las mujeres nativas $(0,201$ y 0,176 puntos logarítmicos en 2006 y 2010, respectivamente). Se observa, además, que ambos diferenciales presentan ciertas variaciones a lo largo de la distribución de salarios. Así, en el caso de la comparación de hombres y mujeres inmigrantes el diferencial presenta un perfil ligeramente decreciente en 2006 y más plano en 2010 (con la excepción de la cola derecha de la distribución, donde crece sustancialmente). Las diferencias salariales entre mujeres nativas e inmigrantes, por su parte, presentan un perfil claramente creciente a lo largo de toda la distribución salarial, algo menos acusado en 2010, que cambia de tendencia únicamente en el extremo superior de la misma. En términos

\footnotetext{
${ }^{6}$ Esta estrategia es equivalente a calcular el promedio de las aportaciones a cada componente de la descomposición de diferentes estimaciones en las que se utilizan alternativamente como referencia cada una de las categorías de cada subconjunto de variables ficticias.
} 


\section{CUADRO 1}

DIFERENCIAS SALARIALES ENTRE HOMBRES INMIGRANTES Y MUJERES INMIGRANTES EN ESPAÑA

\begin{tabular}{|c|c|c|c|}
\hline & $\mathbf{2 0 0 6}$ & $\mathbf{2 0 1 0}$ & Cambio 2006-2010 \\
\hline Promedio & 0,132 & 0,124 & $-0,008$ \\
\hline Percentiles & \multicolumn{3}{|l|}{} \\
\hline 10 & 0,157 & 0,108 & $-0,049$ \\
\hline 20 & 0,152 & 0,109 & $-0,043$ \\
\hline 30 & 0,151 & 0,112 & $-0,039$ \\
\hline 40 & 0,138 & 0,113 & $-0,025$ \\
\hline 50 & 0,133 & 0,108 & $-0,025$ \\
\hline 60 & 0,129 & 0,098 & $-0,031$ \\
\hline 70 & 0,115 & 0,101 & $-0,014$ \\
\hline 80 & 0,110 & 0,104 & $-0,006$ \\
\hline 90 & 0,078 & 0,150 & 0,072 \\
\hline
\end{tabular}

NOTA: La brecha salarial corresponde al diferencial del logaritmo del salario por hora.

\section{CUADRO 2}

DIFERENCIAS SALARIALES ENTRE MUJERES NATIVAS Y MUJERES INMIGRANTES EN ESPAÑA

\begin{tabular}{|c|c|c|c|}
\hline & $\mathbf{2 0 0 6}$ & $\mathbf{2 0 1 0}$ & Cambio 2006-2010 \\
\hline Promedio & 0,201 & 0,176 & $-0,025$ \\
\hline Percentiles & \multicolumn{3}{|l|}{} \\
\hline 10 & 0,039 & 0,084 & 0,045 \\
\hline 20 & 0,068 & 0,094 & 0,026 \\
\hline 30 & 0,100 & 0,124 & 0,024 \\
\hline 40 & 0,127 & 0,153 & 0,026 \\
\hline 50 & 0,176 & 0,192 & 0,016 \\
\hline 60 & 0,250 & 0,220 & $-0,030$ \\
\hline 70 & 0,312 & 0,250 & $-0,062$ \\
\hline 80 & 0,391 & 0,266 & $-0,125$ \\
\hline 90 & 0,376 & 0,262 & $-0,114$ \\
\hline
\end{tabular}

NOTA: La brecha salarial corresponde al diferencial del logaritmo del salario por hora. 


\section{GRÁFICO 1}

BRECHA SALARIAL

HOMBRES Y MUJERES INMIGRANTES

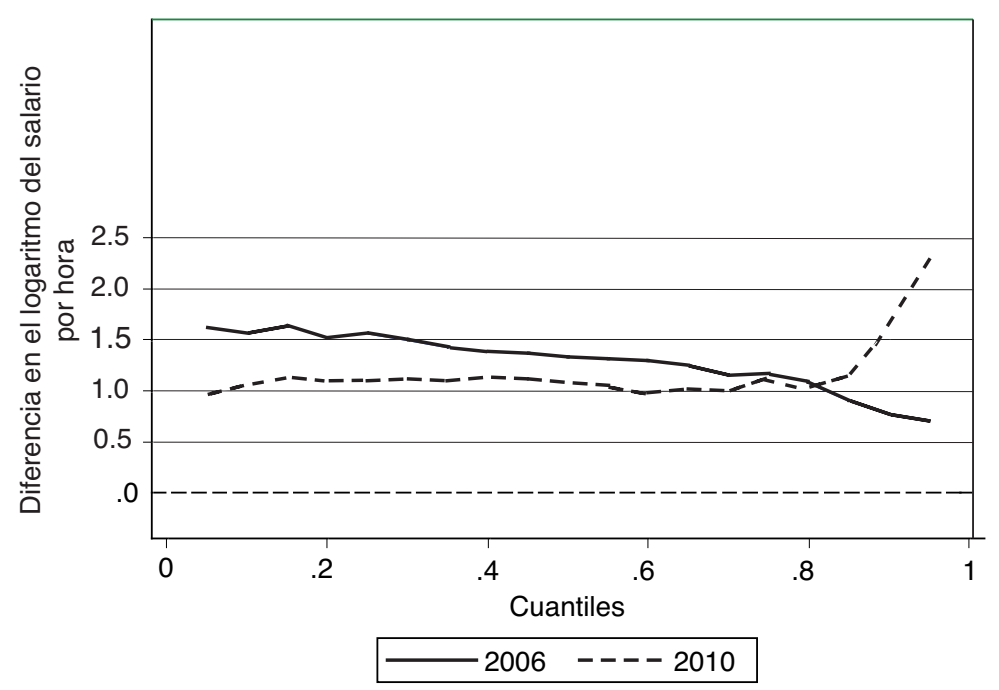

GRÁFICO 2

BRECHA SALARIAL

MUJERES NATIVAS E INMIGRANTES

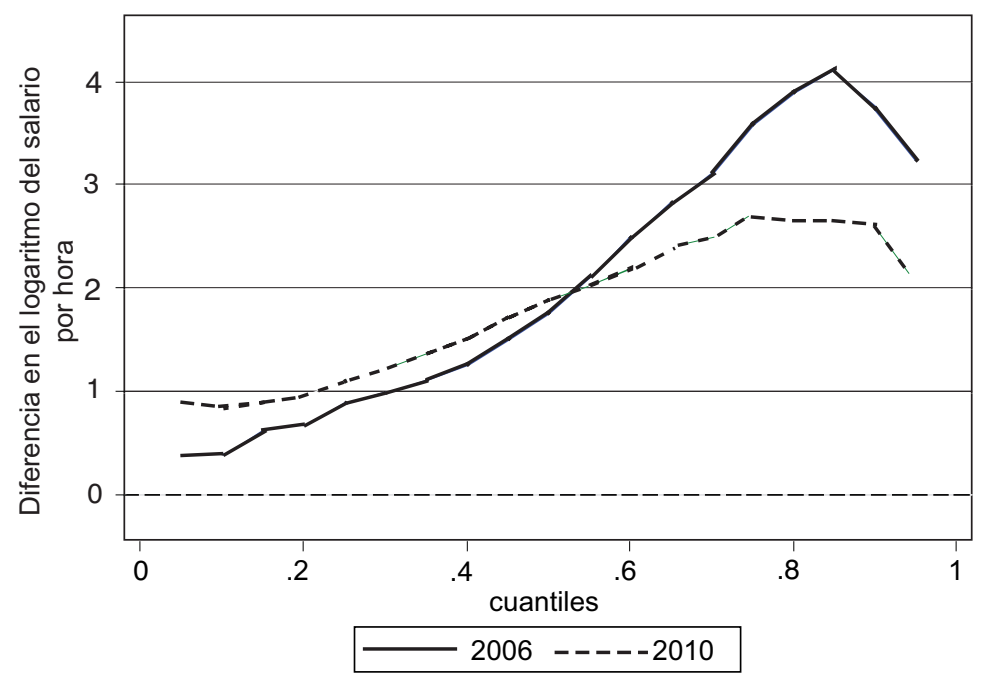


de evolución temporal, por su parte, se observa una reducción de la brecha salarial por género para el colectivo inmigrante, tanto en términos promedio como a lo largo de la distribución de salarios (a excepción de los cuantiles superiores), que contrasta con el aumento de dicho diferencial durante el mismo periodo para el conjunto de la población (Murillo y Simón, 2014). En el mismo sentido, cabe destacar la notable disminución entre 2006 y 2010 del diferencial salarial entre mujeres nativas e inmigrantes, centrada en la mitad superior de la distribución de ingresos. Por el contrario, para las inmigrantes peor posicionadas en términos salariales la desventaja salarial respecto a las nativas aumentó a lo largo del periodo.

El Cuadro A.1 del Anexo muestra, por su parte, la existencia de diferencias significativas en las características relativas de los colectivos considerados. Así, sin ánimo de ser exhaustivos, la comparación entre las mujeres nativas e inmigrantes permite constatar que estas últimas presentan, en promedio, menores niveles de experiencia laboral (aproximada a partir de la edad), educación y antigüedad; una mayor incidencia de los contratos temporales y a tiempo parcial; una mayor presencia en ocupaciones asociadas a menores niveles de cualificación y sin responsabilidades de supervisión y una mayor representación en establecimientos de menor tamaño y cubiertos por convenios de ámbito sectorial. La comparación con los hombres inmigrantes, por su parte, muestra que las mujeres inmigrantes en España son en promedio más jóvenes; presentan mejores niveles educativos; tienen una mayor presencia en puestos de trabajo con contrato indefinido y jornada parcial (pero también en ocupaciones menos cualificadas y sin responsabilidades de supervisión); presentan una distribución sectorial claramente diferenciada (con una elevada presencia relativa de varones en la construcción y de mujeres en sectores como la hostelería o las actividades inmobiliarias y de alquiler) y tienen una mayor presencia en establecimientos de mayor tamaño y cubiertos por convenios de ámbito empresarial.

\subsection{Descomposiciones econométricas}

Los Cuadros 3 y 4 contienen los resultados de la aplicación de la extensión de la metodología de Juhn, Murphy y Pierce $(1991,1993)$ a la descomposición de los diferenciales en salarios medios entre los colectivos comparados en cada uno de los años examinados. La primera fila de cada cuadro contiene el valor del diferencial salarial entre los dos colectivos y el resto el valor de los distintos términos de la descomposición (donde un valor positivo indica que se trata de un factor que origina un diferencial salarial desfavorable para las mujeres inmigrantes frente al otro colectivo). Para cada caso se han considerado tres especificaciones de la ecuación salarial: una que incluye como variables explicativas únicamente características sociodemográficas de los individuos (modelo 1); otra que añade características relativas a puestos de trabajo y establecimientos (modelo 2) y, finalmente, una tercera que incorpora efectos fijos de establecimiento en lugar de los atributos 


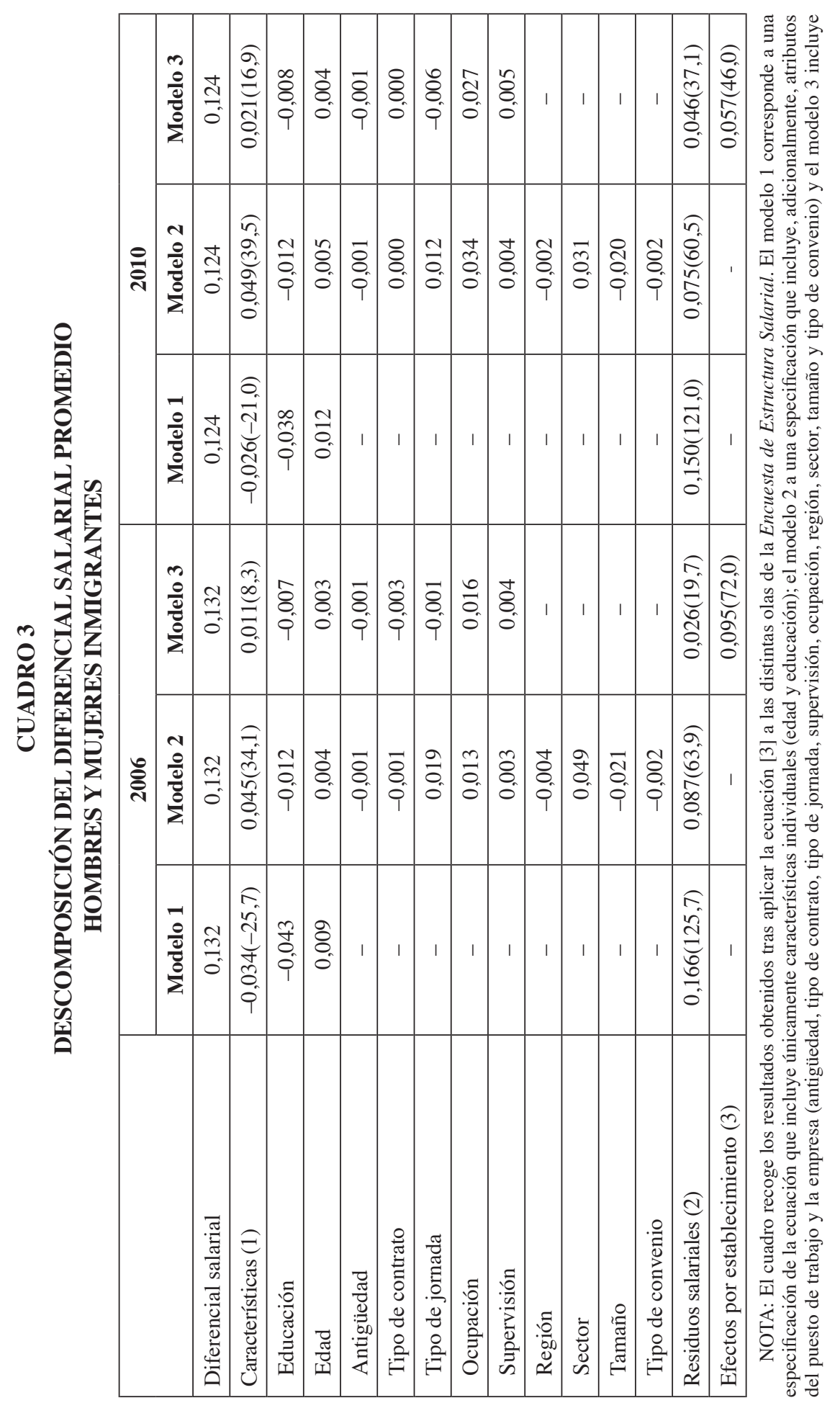




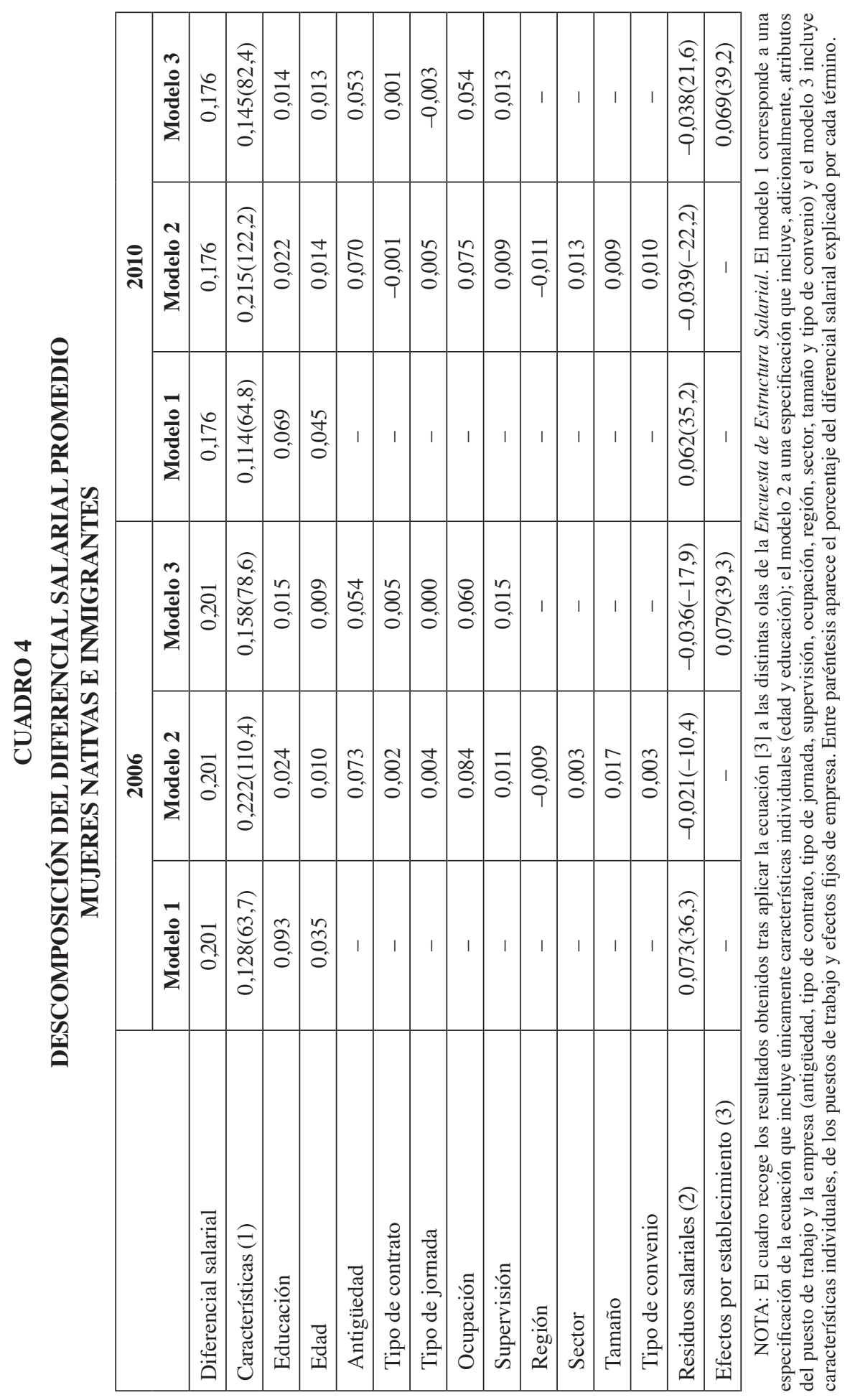


relativos a los mismos. Los resultados de los modelos 1 y 2 son equivalentes a los de una descomposición estándar en dos componentes (características y rendimientos) a la Oaxaca-Blinder, mientras que los del modelo 3 incorporan adicionalmente a los resultados de la descomposición el tercer componente del lado derecho de la ecuación $[3]^{7}$.

En comparación con los hombres inmigrantes, a partir de los resultados del modelo 1 se constata que las mejores dotaciones de niveles educativos de las mujeres inmigrantes determinan que el componente de características tome un valor negativo y que, en consecuencia, todo el diferencial de salarios medios entre los dos colectivos sea atribuible a las diferencias en el tratamiento salarial entre ambos (Cuadro 3). La introducción como variables explicativas adicionales de características de los puestos de trabajo y las empresas (modelo 2) produce ciertas variaciones en los resultados de la descomposición, si bien las diferencias en características continúan explicando una parte menor del diferencial salarial (un 34 por 100 y un 39,5 por 100, en función del año), correspondiendo, en consecuencia, el grueso del mismo (el 63,9 por 100 y 60,5 por 100 restantes) a la parte no explicada.

En cualquier caso, los principales cambios se producen al introducir como variables explicativas los efectos fijos de establecimiento (modelo 3). Las diferencias en las dotaciones de características productivas, capturadas por el primer término de la descomposición, siguen justificando en este caso una parte menor del diferencial de salarios medios entre hombres y mujeres inmigrantes (un 8,3 por 100 en 2006 y un 16,9 por 100 en 2010). Los resultados detallados de la descomposición muestran, en este sentido, que el factor más perjudicial para los salarios relativos de las mujeres inmigrantes es su presencia en ocupaciones con salarios comparativamente reducidos. Los factores inobservables tienen, por su parte, una importante capacidad explicativa del diferencial salarial promedio (el segundo componente de la descomposición toma valores de 0,026 y 0,046 puntos logarítmicos, en función del año, explicando el 19,7 por 100 y el 37,1 por 100 del diferencial salarial). Cabe destacar que, por la naturaleza de la descomposición empleada, el valor de este componente corresponde al diferencial salarial promedio entre hombres y mujeres inmigrantes que trabajan en un mismo establecimiento y que poseen las mismas características productivas observadas. Dicho diferencial es compatible, en consecuencia, con la presencia de mecanismos de discriminación directa por razón de género en los procesos de remuneración salarial del colectivo inmigrante (si bien parte del mismo podría deberse también a factores como la existencia de posibles diferencias en las dotaciones de habilidad inobservada de hombres y mujeres inmigrantes).

Con todo, es la desigual distribución de hombres y mujeres inmigrantes por establecimientos el elemento que explica una mayor proporción del diferencial $(0,095$ y 0,057 puntos logarítmicos, que corresponden a un 72 por 100 y un 46 por 100 del diferencial salarial promedio, en función del año). En consecuencia, cabe concluir

${ }^{7}$ Los resultados detallados de las ecuaciones salariales empleadas en el cálculo de la descomposición están disponibles por parte de los autores ante su requerimiento. 
que la segregación relativa de las mujeres inmigrantes en establecimientos de bajos salarios es un motivo principal de sus menores salarios relativos en relación con los hombres inmigrantes. Los resultados del modelo 2 (donde se incluyen aquellas características de los establecimientos disponibles en la EES) permiten constatar que esta circunstancia está parcialmente relacionada con la mayor presencia relativa de las mujeres inmigrantes en sectores que ofrecen salarios comparativamente reducidos (este factor explicaría por sí solo una proporción de la brecha salarial en torno al 37 por 100 en 2006 y al 25 por 100 en 2010). Por otra parte, resulta llamativo que la relevancia de este elemento se reduzca significativamente entre 2006 y 2010, lo cual puede deberse al distinto impacto sobre ambos colectivos del proceso de destrucción de empleo tras el inicio de la crisis económica.

Cuando la comparación se realiza entre mujeres nativas e inmigrantes (Cuadro 4), los resultados de la descomposición del diferencial de salarios medios muestran que las peores dotaciones de características individuales (educación y edad) de las mujeres inmigrantes determinan que el componente de características explique en torno a dos tercios del diferencial salarial en el modelo 1, por lo que sólo una parte menor del mismo es atribuible, en consecuencia, a las diferencias en el tratamiento salarial entre ambos grupos. Los resultados del modelo 2 muestran, por su parte, que la incorporación de variables relativas a las características de los puestos de trabajo y las empresas provoca que la totalidad del diferencial salarial entre ambos colectivos de mujeres se explique por las diferencias en características, tomando el segundo componente incluso valores negativos. Los resultados de la descomposición detallada muestran, a su vez, que las diferencias en dotaciones de antigüedad y en la distribución ocupacional son dos elementos especialmente relevantes en este sentido, explicando ambos de forma conjunta en torno al 80 por 100 del diferencial en ambos años.

Tras la introducción como variables explicativas de los efectos fijos de establecimiento (modelo 3), las diferencias en dotaciones de características productivas continúan justificando el grueso del diferencial de salarios medios entre mujeres nativas e inmigrantes (en torno a un 80 por 100 en ambos años). Los factores inobservables presentan, al igual que en el modelo 2 , valores negativos $(-0,036$ y $-0,038$, respectivamente), lo que sugiere que las mujeres inmigrantes reciben en promedio un mejor tratamiento salarial que las mujeres nativas con las mismas características productivas observadas que trabajan en su mismo establecimiento ${ }^{8}$. Finalmente, al igual que en la comparación por género, la desigual distribución de ambos colectivos por establecimientos es un factor con una influencia significativa en el diferencial salarial, explicando en torno al 40 por 100 del mismo en ambos años.

Desde una perspectiva temporal, el Cuadro 5 muestra los resultados de la descomposición del cambio experimentado por el diferencial salarial entre cada par de colectivos entre 2006 y 2010. Dichos resultados han sido obtenidos mediante la

${ }^{8}$ SIMÓN et al. (2008a) también documentan con datos relativos a la ola de 2002 de la EES y una descomposición salarial similar a la aquí planteada un efecto favorable a las mujeres inmigrantes frente a las nativas de los factores inobservables. 
ecuación [4] y la inclusión de efectos fijos de empresa en la especificación de la ecuación salarial. La primera fila del cuadro contiene el valor del cambio experimentado por el diferencial salarial con el transcurso del tiempo, mientras que las filas restantes muestran la contribución de los distintos términos de la descomposición (donde un valor positivo indica que el correspondiente factor contribuye a un aumento del diferencial salarial).

\section{CUADRO 5}

DESCOMPOSICIÓN DEL CAMBIO EN EL DIFERENCIAL SALARIAL PROMEDIO ENTRE 2006 Y 2010. HOMBRES Y MUJERES INMIGRANTES Y MUJERES NATIVAS E INMIGRANTES

\begin{tabular}{|l|c|c|}
\hline & $\begin{array}{c}\text { Hombres-mujeres } \\
\text { inmigrantes }\end{array}$ & $\begin{array}{c}\text { Mujeres-nativas } \\
\text { inmigrantes }\end{array}$ \\
\hline Diferencial salarial 2010-Diferencial salarial 2006 & $-0,008$ & $-0,025$ \\
\hline Dotación relativa de características (1) & $0,010(-125,0)$ & $-0,017(68,0)$ \\
\hline Educación & 0,002 & $-0,006$ \\
\hline Edad & 0,001 & 0,001 \\
\hline Antigüedad & 0,000 & $-0,001$ \\
\hline Tipo de contrato & 0,000 & 0,000 \\
\hline Tipo de jornada & 0,001 & $-0,001$ \\
\hline Ocupación & 0,004 & $-0,007$ \\
\hline Supervisión & 0,002 & $-0,002$ \\
\hline Rendimientos de las características (2) & $0,001(-12,5)$ & $0,004(-16,0)$ \\
\hline Educación & $-0,004$ & 0,005 \\
\hline Edad & 0,000 & 0,002 \\
\hline Antigüedad & 0,000 & 0,000 \\
\hline Tipo de contrato & 0,002 & $-0,004$ \\
\hline Tipo de jornada & $-0,006$ & $-0,001$ \\
\hline Ocupación & 0,007 & 0,002 \\
\hline Supervisión & 0,000 & 0,000 \\
\hline Residuos salariales relativos (3) & $0,019(-237,5)$ & $-0,002(8,0)$ \\
\hline Dispersión de los residuos salariales (4) & $0,000(0,0)$ & $0,000(0,0)$ \\
\hline Efectos fijos por establecimiento relativos (5) & $-0,039(487,5)$ & $-0,011(44,0)$ \\
\hline Dispersión de los efectos fijos por establecimiento (6) & $0,001(-12,5)$ & $0,001(-4,0)$ \\
\hline
\end{tabular}

NOTA: El cuadro recoge los resultados obtenidos tras aplicar la ecuación [4] a las olas de 2006 y 2010 de la Encuesta de Estructura Salarial. El modelo utilizado corresponde a una especificación de la ecuación que incluye efectos fijos de empresa. Entre paréntesis aparece el porcentaje de cambio en el diferencial salarial por razón de género explicado por cada término. 
A partir de dicha evidencia se constata que la ligera reducción experimentada entre 2006 y 2010 por la brecha salarial entre hombres y mujeres inmigrantes $(-0,008$ puntos logarítmicos) se debe al efecto contrapuesto de diversos factores. Así, por una parte, la reducción de la intensidad de la segregación de este colectivo en establecimientos de bajos salarios relativos tuvo un notable efecto positivo sobre los salarios relativos de las mujeres inmigrantes (el quinto componente de la descomposición toma un valor de $-0,039$ puntos logarítmicos). No obstante, el mismo resultó en buena medida contrarrestado por el efecto perjudicial derivado tanto del empeoramiento de su dotación relativa de características (con un papel destacado de los cambios en la distribución por ocupaciones) como, muy especialmente, del empeoramiento durante el periodo de su posición en la distribución de residuos salariales (el primer y el tercer componente de la descomposición toman valores de 0,010 y 0,019 puntos logarítmicos, respectivamente). A su vez, la reducción más significativa observada en el periodo en la brecha salarial entre las mujeres nativas e inmigrantes $(-0,025$ puntos logarítmicos) se explica esencialmente por la influencia conjunta de la mejora en la dotación relativa de las características productivas de las mujeres inmigrantes (el primer componente de la descomposición toma un valor de $-0,017$ puntos logarítmicos $\mathrm{y}$, al igual que en el caso anterior, de la menor intensidad de la segregación de las mujeres en establecimientos de bajos salarios relativos (el quinto componente toma un valor de $-0,011$ puntos).

En otro orden de cosas, los Gráficos 3 a 6 contienen los resultados de la descomposición de la diferencia por cuantiles del logaritmo del salario por hora entre los colectivos comparados, obtenidos tras aplicar la metodología propuesta por Fortin, Lemieux y Firpo (2011). Con el fin de facilitar la presentación, los Gráficos 3 y 4 distinguen únicamente entre la aportación agregada de los componentes de características y de rendimientos, mientras que los Gráficos 5 y 6 contienen los resultados detallados de los efectos individuales de las variables explicativas asociados al componente de características ${ }^{9}$. Esta evidencia ha sido obtenida, a su vez, utilizando dos conjuntos diferentes de variables explicativas: uno que incluye únicamente características sociodemográficas de los individuos (modelo 1) y otro que añade características relativas a puestos de trabajo y establecimientos (modelo 2). En este último caso, también con el objetivo de facilitar la presentación, dichas variables han sido agrupadas en tres categorías, en función de si se trata de características individuales de los trabajadores, de sus puestos de trabajo o de sus empresas.

Así, los resultados agregados obtenidos con el modelo 1 (donde se contemplan como variables explicativas exclusivamente la educación y la edad) muestran que el diferencial salarial entre hombres y mujeres inmigrantes se explica a lo largo de toda la distribución salarial en su práctica totalidad por las diferencias en rendimientos

\footnotetext{
${ }_{9}^{9}$ Puede encontrarse información adicional sobre los resultados de la descomposición en los Cuadros A.2 a A.9 del Anexo. La información relativa a los coeficientes estimados mediante el método de regresión cuantílica incondicional sobre el que se fundamenta la metodología de descomposición está disponible por parte de los autores ante su requerimiento.
} 
(Gráfico 3). Consecuentemente, el componente de características presenta una aportación negligible en la parte baja de la distribución, resultando incluso favorable para las mujeres inmigrantes a medida que se avanza a lo largo de la misma. Este último resultado se explica, a su vez, esencialmente por las diferencias en las dotaciones de educación de ambos colectivos (gráfico 5). Cabe destacar, asimismo, que esta evidencia es consecuente, en general, con la obtenida con la descomposición de los salarios medios de los dos colectivos comparados (Cuadro 3).

La introducción de las características de los puestos de trabajo y de las empresas como variables explicativas (modelo 2) produce modificaciones reseñables en los resultados de la descomposición. Así, en primer lugar, las diferencias en dotaciones de características continúan siendo favorables para las mujeres inmigrantes únicamente en la parte superior de la distribución salarial, resultando perjudiciales en la parte baja de la misma. La evidencia detallada revela, por su parte, que esta última circunstancia se debe en buena medida a las diferencias en dotaciones de características de las empresas (y, en particular, de las diferencias en la distribución sectorial), mientras que el efecto positivo de las características en la parte superior de la distribución se explica por diferencias en dotaciones de características de los puestos de trabajo, con especial relevancia para la ocupación y el tipo de jornada (Gráfico 5 y Cuadros A.6 y A.7). En relación con el efecto de los coeficientes, al igual que en el modelo 1, es sistemáticamente desfavorable para las mujeres inmigrantes, observándose ahora un perfil claramente creciente para el componente de rendimientos a lo largo de la distribución salarial en los dos años considerados.

Por su parte, los resultados agregados obtenidos con el modelo 1 revelan que el diferencial salarial entre mujeres nativas e inmigrantes (Gráfico 4) se explica en mayor medida por el componente de características que por el de rendimientos a lo largo de toda la distribución salarial (con la única excepción de la cola derecha en el año 2006), así como que el principal factor explicativo relacionado con las características individuales son las diferencias en las dotaciones de educación (Gráfico 6 y Cuadros A.2 y A.3). No obstante, en concordancia con la evidencia obtenida tras la descomposición de las diferencias en salarios medios, la introducción de las características de los puestos de trabajo y de las empresas como variables explicativas (modelo 2) produce modificaciones significativas en los resultados de la descomposición. Así, el componente de rendimientos toma valores sistemáticamente negativos a lo largo de toda la distribución salarial (con las únicas excepciones de los cuantiles superiores de la distribución en 2006 y el cuantil inferior en 2010), lo que implica que el diferencial salarial entre mujeres nativas e inmigrantes se explica en su totalidad por las diferencias en sus dotaciones de características (Gráfico 4). Cabe destacar, por último, que resultan especialmente relevantes en este sentido las diferencias en dotaciones de características de los puestos de trabajo, con especial influencia de la antigüedad y la ocupación (Gráfico 6 y Cuadros A.8 y A.9). 

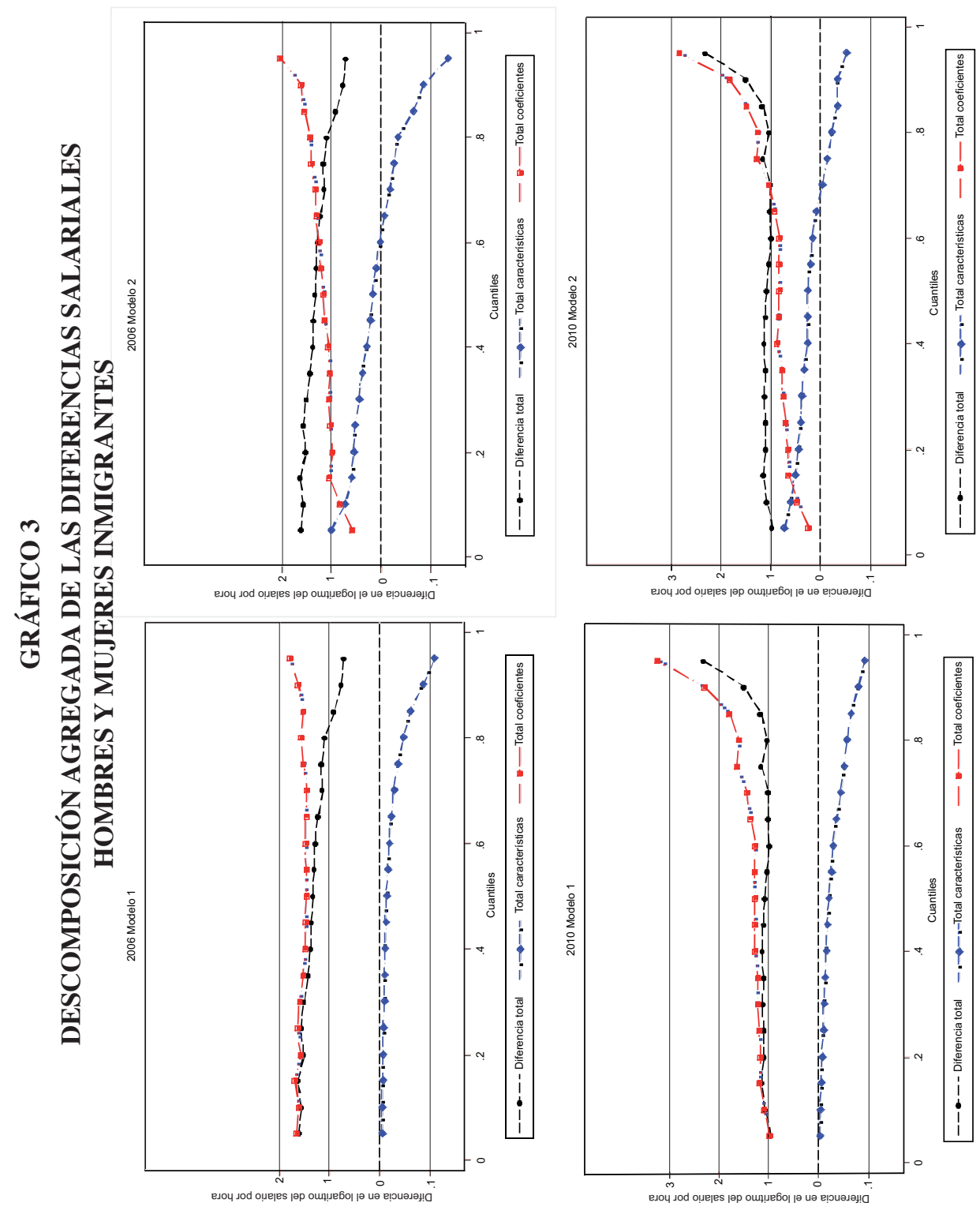

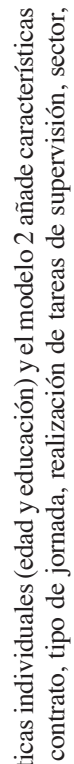

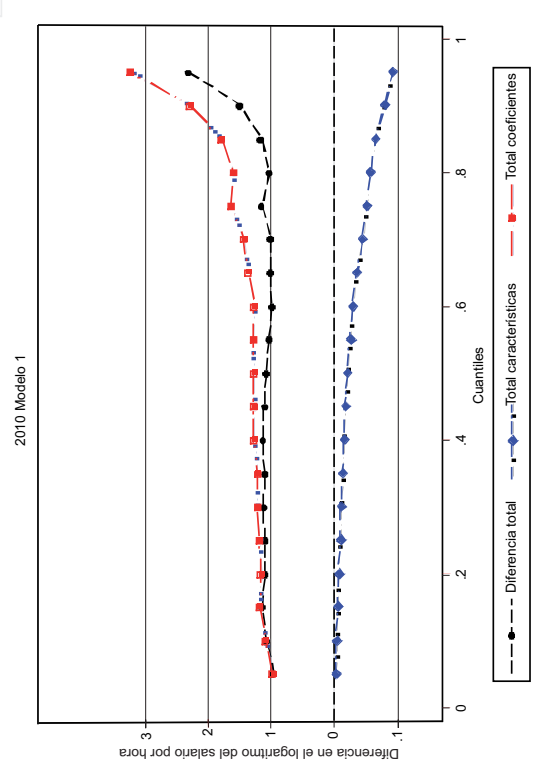

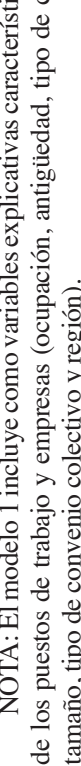



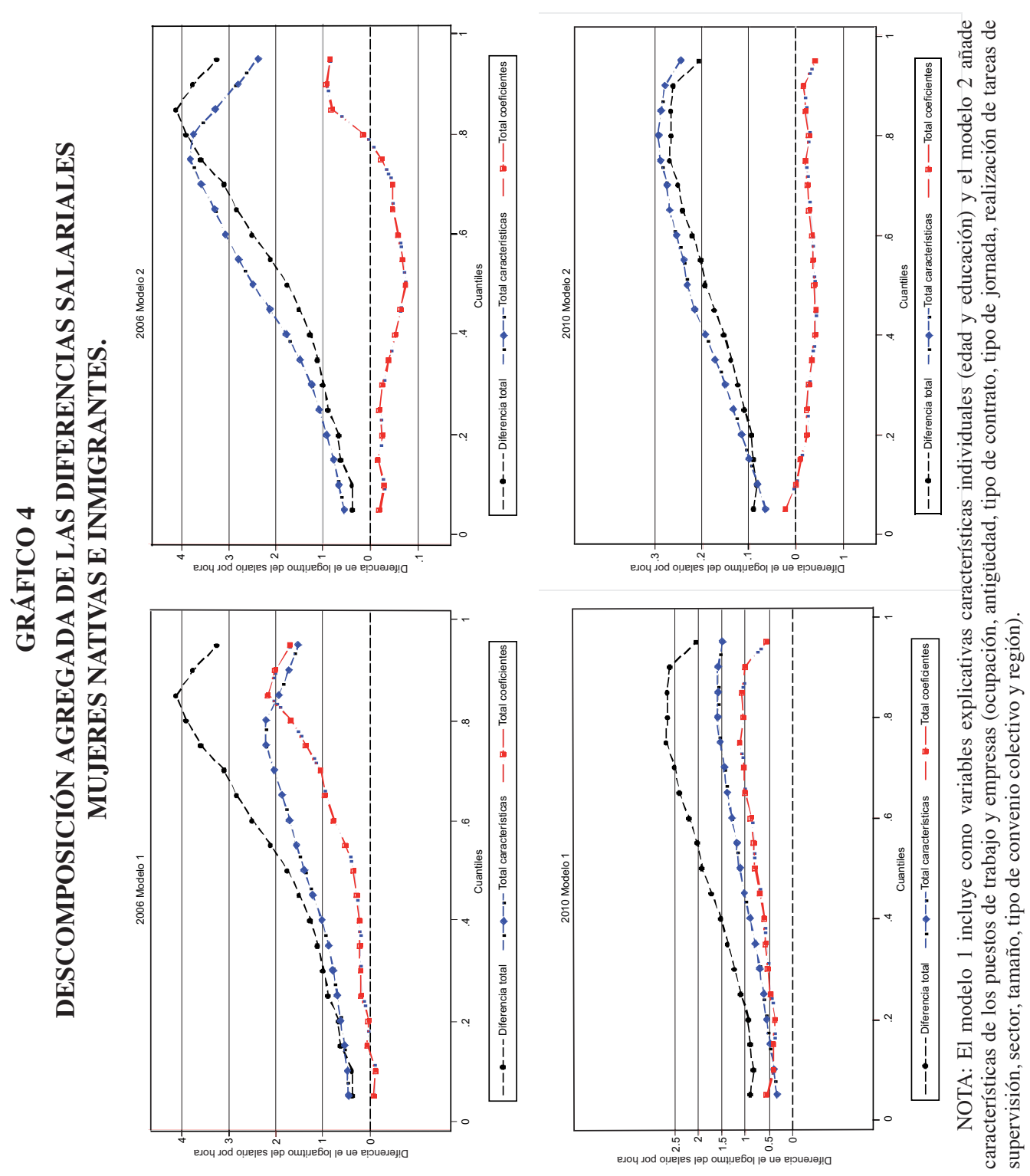


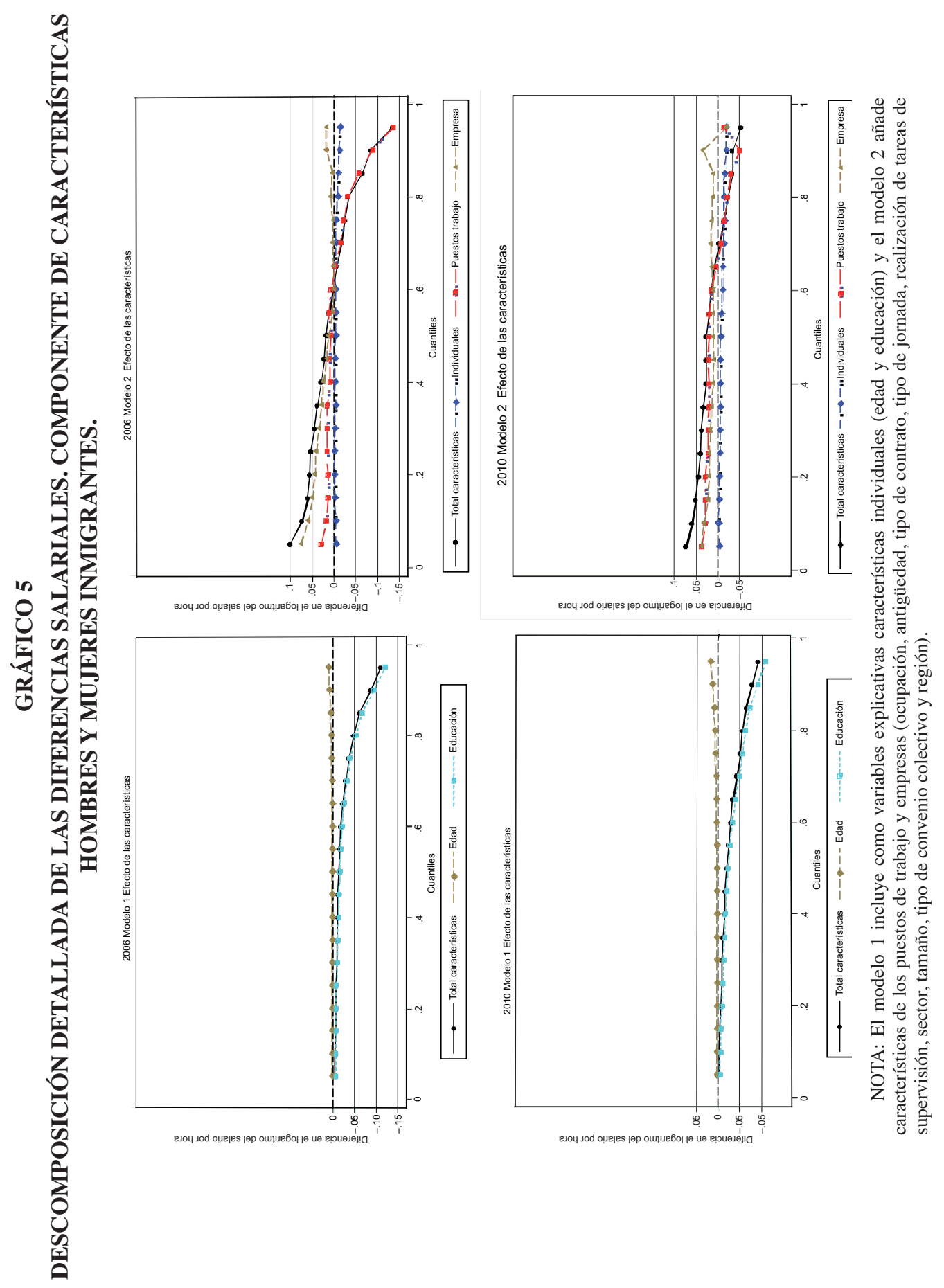




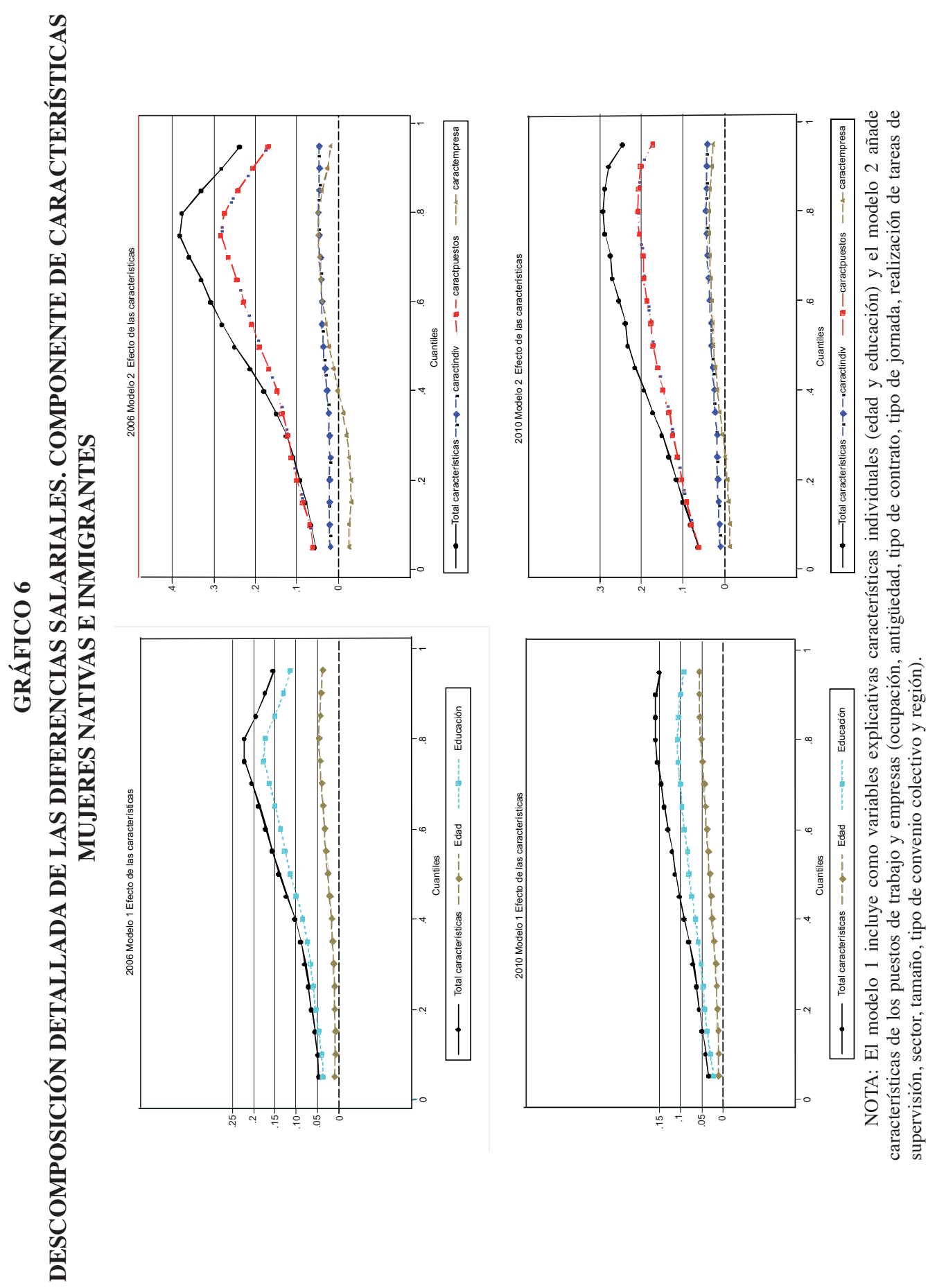




\section{Conclusiones}

Esta investigación examina los salarios relativos de las mujeres inmigrantes en España a partir de las olas más recientes de la Encuesta de Estructura Salarial con la finalidad principal de contrastar si este colectivo sufre una doble penalización salarial en relación con los hombres inmigrantes y las mujeres nativas. El análisis empírico se desarrolla a partir de la aplicación de dos técnicas econométricas de descomposición de las diferencias salariales: una extensión de la metodología de Juhn, Murphy y Pierce $(1991,1993)$ adaptada a su uso con microdatos emparejados empresa-trabajador que permite considerar, entre otros aspectos, el efecto del establecimiento del trabajador sobre la brecha salarial promedio y su evolución en el tiempo y una técnica propuesta por Fortin, Lemieux y Firpo (2011) que posibilita desarrollar de forma detallada el análisis de las diferencias salariales a lo largo de la distribución de salarios.

La evidencia obtenida confirma que en el mercado de trabajo español las mujeres inmigrantes presentan desventajas salariales significativas frente a los hombres inmigrantes y a las mujeres nativas. Estas últimas son algo más acusadas y presentan, en general, un perfil claramente creciente a lo largo de la distribución salarial. La desventaja salarial de las mujeres inmigrantes muestra, no obstante, una tendencia a la reducción a lo largo del periodo considerado, observándose con el transcurso del tiempo una mejora relativa de los salarios relativos de las mismas tanto en comparación con los varones inmigrantes como, sobre todo, con las mujeres nativas. Esta tendencia que se constata para los salarios promedio se da, sin embargo, exclusivamente para el colectivo situado en la parte alta de la distribución salarial, ya que para las mujeres inmigrantes peor posicionadas se observa únicamente una mejora de la brecha salarial por género, que contrasta con un empeoramiento en el diferencial salarial respecto a las mujeres nativas.

Los resultados de los ejercicios de descomposición permiten constatar que las significativas diferencias que se observan en las características relativas son un origen importante de los salarios comparativamente menores de las mujeres inmigrantes, tanto frente a las mujeres nativas como frente a los hombres inmigrantes. No obstante, mientras que la desventaja salarial frente a las mujeres nativas resulta explicada en su totalidad por las diferencias en características productivas, en relación con los varones inmigrantes destaca la presencia de una significativa brecha salarial originada por una distinta remuneración de las características observadas. Cabe destacar, además, que la misma tiene un carácter intraempresa, lo que refuerza la conclusión de que ambos colectivos reciben, en general, un tratamiento salarial diferenciado.

En otro orden de cosas, los resultados obtenidos muestran que entre las características observadas de las mujeres inmigrantes con un efecto cuantitativamente más pernicioso sobre sus salarios relativos destacan factores como la ocupación, el sector y, en general, el establecimiento de pertenencia (y, en el caso concreto de la comparación con las mujeres nativas, también la antigüedad). En el mismo sentido, desde una perspectiva temporal se constata que uno de los elementos 
más determinantes en la evolución de su brecha salarial frente a otros colectivos son los cambios en la intensidad de la segregación de las mujeres inmigrantes en establecimientos de bajos salarios relativos. Esta evidencia confirma, en suma, que la segregación de este colectivo en segmentos del mercado de trabajo caracterizados por ofrecer salarios comparativamente bajos es un elemento explicativo fundamental de sus menores salarios relativos. Se trata de un resultado coincidente con los hallazgos de estudios previos para España que justifican el papel determinante de la segregación laboral de las mujeres y de los inmigrantes sobre este fenómeno (Amuedo-Dorantes y De la Rica, 2006; Simón et al., 2008a,b; García-Pérez et al., 2012; Nicodemo y Ramos, 2012).

Para concluir, cabe destacar que la evidencia obtenida en la presente investigación para España no concuerda plenamente con la hipótesis de la existencia de una doble penalización salarial para las mujeres inmigrantes. Así, si bien en el caso español los menores salarios de las mujeres inmigrantes en comparación con los hombres inmigrantes se deben en parte a un peor tratamiento salarial, las mejores dotaciones productivas de las mujeres nativas parecen justificar en gran medida sus mayores salarios relativos. En consecuencia, los resultados obtenidos para nuestro país discrepan de los documentados para otras economías avanzadas donde a la penalización salarial por razón de género las mujeres inmigrantes añaden la derivada de su condición de extranjera (Shamsuddin, 1998; Hayfron, 2001).

\section{Referencias bibliográficas}

[1] ADSERA, A. y CHISWICK, B. R. (2007): «Are there gender and country of origin differences in immigrant labor market outcomes across European destinations?», Journal of Population Economics, vol. 20, pp. 495-526.

[2] ALCOBENDAS, M. A. y RODRÍGUEZ-PLANAS, N. (2009): «Immigrant assimilation process in a segmented labor market», IZA Discussion Paper núm. 4394.

[3] ALONSO-VILLAR, O. y DEL RÍO, C. (2013): «Occupational segregation in a country of recent mass immigration: Evidence from Spain», Annals of Regional Science, vol. 50 (1), pp. 109-134.

[4] ALTONJI, J. G. y BLANK, R. (1999): «Race and gender in the labor market», en O. Ashenfelter y D. Card (eds.), Handbook of Labour Economics, vol. 3, Amsterdam: North-Holland, pp. 3143-3259.

[5] AMUEDO-DORANTES, C. y DE LA RICA, S. (2006): «The role of segregation and pay structure on the gender wage gap: Evidence from matched employer-employee data for Spain», Contributions to Economic Analysis and Policy, Berkley Electronic Press Journals.

[6] AMUEDO-DORANTES, C. y DE LA RICA, S. (2007): «Labour market assimilation of recent immigrants in Spain», British Journal of Industrial Relations, vol. 45 (2), pp. 257-284.

[7] AMUEDO-DORANTES, C. y DE LA RICA, S. (2011): «Complements or substitutes? Task specialization by gender and nativity in Spain», Labour Economics, vol. 18, pp. 697-707. 
[8] ANTECOL, H.; COBB-CLARK, D. y TREJO, S. (2003): «Immigration policy and the skills of immigrants to Australia, Canada and the United States», Journal of Human Resources, vol. 38 (1), pp. 192-218.

[9] BAKER, M. y BENJAMIN, D. (1997): «The role of the family in immigrants' labormarket activity: an evaluation of alternative explanations», American Economic Review, vol. 87 (4), pp. 705-727.

[10] BEACH, C. y WORSWICK, C. (1993): «Is there a double-negative effect on the earnings of immigrant women?», Canadian Public Policy, vol. 19 (1), pp. 36-53.

[11] CARLINER, G. (1980): «Wages, earnings and hours of first, second and third generation American males», Economic Inquiry, vol. 18 (1), pp. 87-102.

[12] COBB-CLARK, D. A. y CROSSEY, T. F. (2004): «Revisting the family investment hypothesis», Labour Economics, vol. 11 (3), pp. 373-393.

[13] CONSTANT, A. F. y ZIMMERMANN, K. F. (2013): «Migration and ethnicity: an introduction», en International Handbook on the Economics of Migration, Edwar Elgan, Cheltenham, UK.

[14] CHISWICK, B. R. (1978): «The effect of Americanization on the earnings of foreignborn men», Journal of Political Economy, vol. 86 (5), pp. 897-922

[15] DE LA RICA, S.; DOLADO, J. J. y VEGAS, R. (2010): «Performance pay and the gender wage gap: Evidence for Spain», IZA Discussion Paper 5032.

[16] DEL RÍO, C. y ALONSO-VILLAR, O. (2012): «Occupational segregation of immigrant women in Spain», Feminist Economics, vol. 18 (2), pp. 91-123.

[17] DI NARDO, J.; FORTIN, N. M. y LEMIEUX, T. (1996): «Labor market institutions and the distribution of wages, 1973-1992: A semi-parametric approach», Econometrica, 64 (5), pp. 1011-1044.

[18] DULEEP, H. y SANDERS, S. (1993): «The decision to work by married immigrant women: evidence from Asian women», Industrial and Labor Relations Review, vol. 46 (4), pp. 677-690.

[19] DUSTMANN, C. y SCHMIDT, C. (2000): «The wage performance of immigrant women: full-time jobs, part-time jobs, and the role of selection», IZA Discussion Paper n. ${ }^{\circ} 233$.

[20] FIRPO, S.; FORTIN, N. y LEMIEUX, T. (2009): «Unconditional Quantile Regressions», Econometrica, 77(3), pp. 953-973.

[21] FORTIN, N.; LEMIEUX, T. y FIRPO, S. (2011): «Decomposition Methods in Economics», Handbook of Labor Economics, vol. 4, cap. 1, pp. 1-102. Elsevier.

[22] GARCÍA PÉREZ, J. I.; MUÑOZ BULLÓN, F. y PRIETO RODRÍGUEZ, M. (2012): «The wage gap between foreign and Spanish nationals in Spain: an analysis using matched employer-employee data», International Migration. doi: 10.1111/imig.12000

[23] HAYFRON, J. E. (2001): «Panel estimates of the earnings gap in Norway: do female immigrants experience a double earnings penalty?», Applied Economics, vol. 34, pp. 1441-1452.

[24] HECKMAN, J. (1979): «Sample Selection Bias as a Specification Error», Econometrica, 47(1), pp. 153-161.

[25] JUHN, C.; MURPHY, K. y PIERCE, B. (1991): «Accounting for the Slowdown in Black-White Convergence», en M. Osters (ed.) Workers and Their Wages, 107-143. Washington DC: American Enterprise Institute Press.

[26] JUHN, C.; MURPHY, K. y PIERCE, B. (1993): «Wage inequality and the rise in returns to skill», Journal of Political Economy, vol. 101, pp. 410-442. 
[27] LECLERE, F. y MCLAUGHLIN, D. (1997): «Family migration and changes in women's earnings: A decomposition analysis», Population Research and Policy Review, vol. 16 (4), pp. 315-335.

[28] LONG, J. E. (1980): «The effect of the Americanization on earnings: Some evidence for women», Journal of Political Economy, vol. 88 (3), pp. 620-629.

[29] MACHADO, J. y MATA, J. A. F. (2005): «Conterfactual decomposition of changes in wage distributions using quantile regression», Journal of Applied Econometrics, 20 (4), pp. 445-465.

[30] MACPHERSON, D. A. y STEWART, J. B. (1989): «The labor force participation and earnings profile of married female immigrants», Quarterly Review of Economics and Business, vol. 29(2), pp. 57-72.

[31] MELLY, B. (2005): «Decomposition of differences in distribution using quantile regression», Labour Economics, Elsevier, 12 (4), pp. 577-590.

[32] MELLY, B. (2006): «Estimation of counterfactual distributions using quantile regression», mimeo, Swiss Institute for International Economics and Applied Economic Research, University of St. Gallen.

[33] MINCER, J. (1978): «Family migration decisions», Journal of Political Economy, vol. 86 (5), pp. 749-773.

[34] MURILLO, I. P. y SIMÓN, H. (2014): «La Gran Recesión y el diferencial salarial por género: evidencia para España», Hacienda Pública Española, de próxima publicación.

[35] NEUMARK, D. (1988): «Employer's discriminatory behaviour and the estimation of wage discrimination», Journal of Human Resources, vol. 23, pp. 279-295.

[36] NICODEMO, C. and RAMOS, R. (2012): «Wage differentials between native and immigrant women in Spain. Accounting for differences in support», International Journal of Manpower, vol. 33 (1), pp. 118-136.

[37] OAXACA, R. y RANSOM, M. (1994): «On discrimination and the decomposition of wage differentials», Journal of Econometrics, 61, pp. 5-22.

[38] OAXACA,R. y RAMSON,M.(1999): «Identification in detailed wage decompositions», The Review of Economics and Statistics, 81 (1), pp. 154-157.

[39] RAMOS, R.; SANROMÁ, E. y SIMÓN, H. (2014): «Public-private sector wage differentials by type of contract: Evidence from Spain», Hacienda Pública Española (aceptado para publicación).

[40] REIMERS, C. W. (1985): «Cultural differences in labor force participation among married women», American Economic Review, vol. 75 (2), págs. 251-255.

[41] SHAMSUDDIN, A. F. (1998): «The double-negative effect on the earnings of foreignborn females in Canada», Applied Economics, vol. 30, pp. 1187-1201.

[42] SIMÓN, H.; RAMOS, R. y SANROMÁ, E. (2008a): «Labour Segregation and Immigrant and Native-born Wage Distributions in Spain: An Analysis Using Matched Employer-Employee Data», Spanish Economic Review, vol. 10 (2), pp. 135-168.

[43] SIMÓN, H.; RAMOS, R. y SANROMÁ, E. (2008b): «Evolución de las diferencias salariales por razón de sexo», Revista de Economía Aplicada, vol. XVI (48), pp. 37 68.

[44] YUN, M. (2005): «A Simple Solution to the Identification Problem in Detailed Wage Decompositions», Economic Inquiry, vol. 43, pp. 766-772. 


\begin{abstract}
ANEXO
GRÁFICO A.1

DISTRIBUCIÓN SALARIAL DE HOMBRES Y MUJERES INMIGRANTES
\end{abstract}

(2006 y 2010)

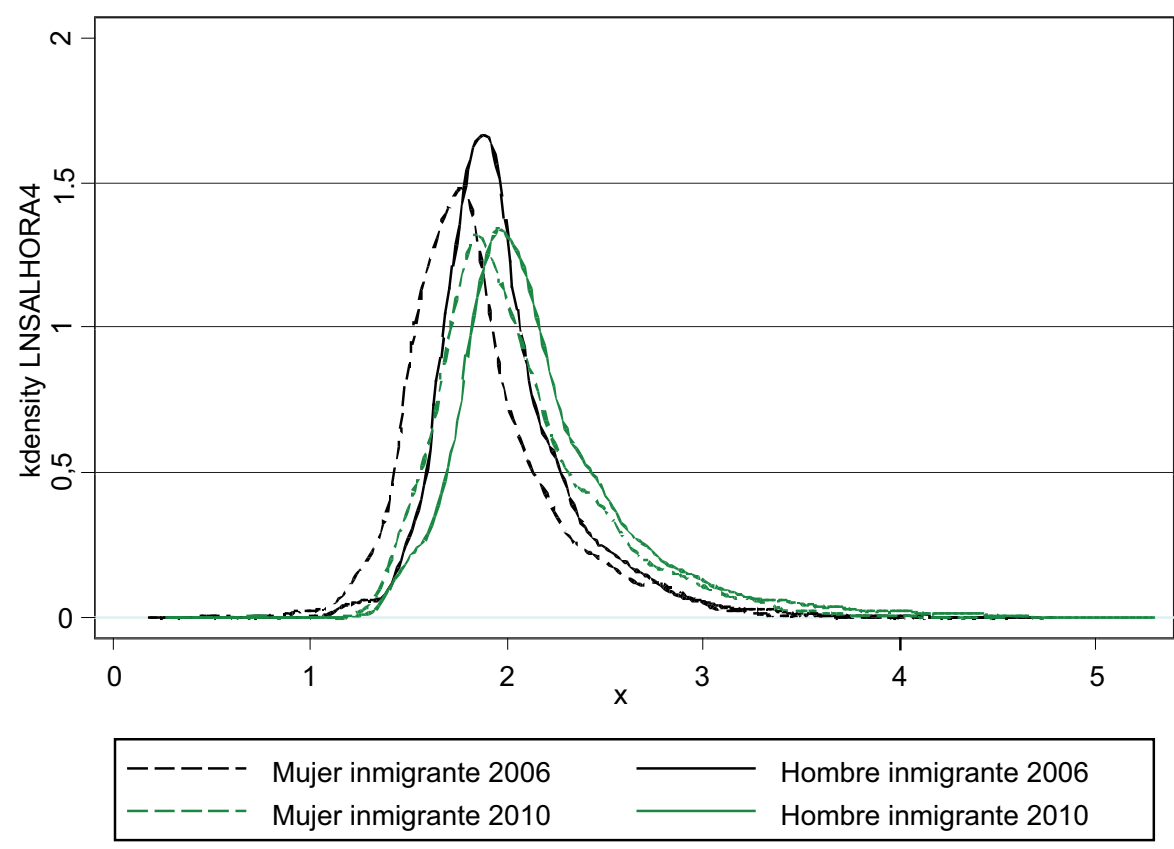

NOTA: En el gráfico aparece la función de densidad del logaritmo del salario por hora. 
GRÁFICO A.2

DISTRIBUCIÓN SALARIAL DE MUJERES NATIVAS E INMIGRANTES (2006 y 2010)

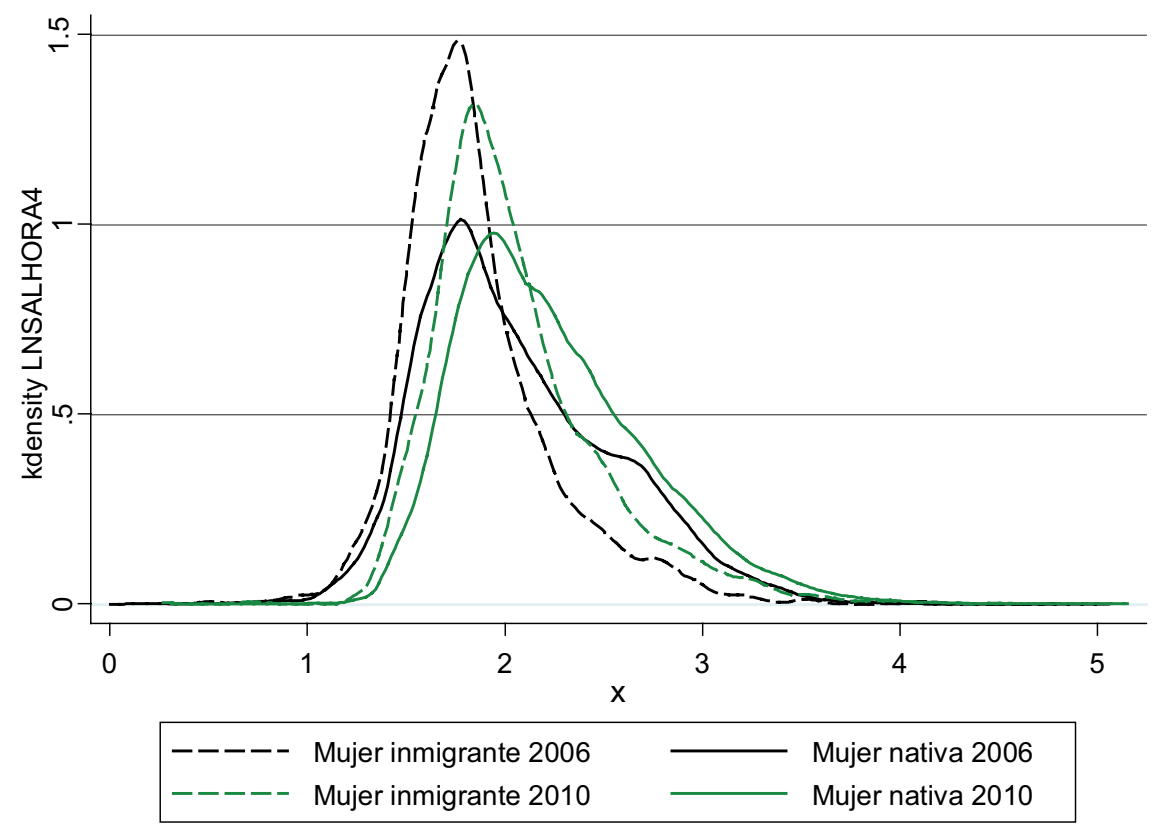

NOTA: En el gráfico aparece la función de densidad del logaritmo del salario por hora. 
CUADRO A.1

DESCRIPTIVOS DE LAS VARIABLES

\begin{tabular}{|c|c|c|c|c|c|c|}
\hline & \multicolumn{3}{|c|}{2006} & \multicolumn{3}{|c|}{2010} \\
\hline & $\begin{array}{l}\text { Mujer } \\
\text { nativa }\end{array}$ & $\begin{array}{l}\text { Hombre } \\
\text { inmigrante }\end{array}$ & $\begin{array}{c}\text { Mujer } \\
\text { inmigrante }\end{array}$ & $\begin{array}{l}\text { Mujer } \\
\text { nativa }\end{array}$ & \begin{tabular}{|c|} 
Hombre \\
inmigrante
\end{tabular} & $\begin{array}{c}\text { Mujer } \\
\text { inmigrante }\end{array}$ \\
\hline Salario por hora & $\begin{array}{l}8,979 \\
(5,61) \\
\end{array}$ & $\begin{array}{l}8,023 \\
(5,07) \\
\end{array}$ & $\begin{array}{l}7,092 \\
(4,73) \\
\end{array}$ & $\begin{array}{l}10,610 \\
(6,65)\end{array}$ & $\begin{array}{l}10,381 \\
(9,10)\end{array}$ & $\begin{array}{l}8,788 \\
(5,82) \\
\end{array}$ \\
\hline $\begin{array}{l}\text { Logaritmo del } \\
\text { salario por hora }\end{array}$ & $\begin{array}{l}2,060 \\
(0,49)\end{array}$ & $\begin{array}{l}1,991 \\
(0,38) \\
\end{array}$ & $\begin{array}{l}1,859 \\
(0,40) \\
\end{array}$ & $\begin{array}{l}2,234 \\
(0,47)\end{array}$ & $\begin{array}{l}2,182 \\
(0,48)\end{array}$ & $\begin{array}{l}2,058 \\
(0,43) \\
\end{array}$ \\
\hline Inmigrante: Europa & - & 0,358 & 0,333 & - & 0,437 & 0,460 \\
\hline $\begin{array}{l}\text { Inmigrante: } \\
\text { América Latina }\end{array}$ & - & 0,346 & 0,538 & - & 0,322 & 0,447 \\
\hline $\begin{array}{l}\text { Inmigrante: resto } \\
\text { del mundo }\end{array}$ & - & 0,296 & 0,128 & - & 0,241 & 0,093 \\
\hline Edad menos de 30 & 0,254 & 0,287 & 0,334 & 0,183 & 0,223 & 0,270 \\
\hline Edad 30-45 & 0,492 & 0,576 & 0,530 & 0,538 & 0,617 & 0,581 \\
\hline Edad más de 45 & 0,254 & 0,137 & 0,136 & 0,278 & 0,159 & 0,148 \\
\hline Educación primaria & 0,207 & 0,542 & 0,428 & 0,115 & 0,357 & 0,248 \\
\hline Educación secundaria & 0,499 & 0,372 & 0,422 & 0,568 & 0,484 & 0,545 \\
\hline Educación terciaria & 0,294 & 0,085 & 0,150 & 0,316 & 0,159 & 0,207 \\
\hline Antigüedad & $\begin{array}{l}6,647 \\
(8,55) \\
\end{array}$ & $\begin{array}{l}1,243 \\
(2,78)\end{array}$ & $\begin{array}{l}1,320 \\
(2,91) \\
\end{array}$ & $\begin{array}{l}7,815 \\
(8,71) \\
\end{array}$ & $\begin{array}{l}2,375 \\
(3,04)\end{array}$ & $\begin{array}{l}2,419 \\
(3,06)\end{array}$ \\
\hline Contrato temporal & 0,278 & 0,580 & 0,467 & 0,221 & 0,395 & 0,333 \\
\hline Contrato indefinido & 0,722 & 0,420 & 0,533 & 0,779 & 0,605 & 0,667 \\
\hline Jornada parcial & 0,278 & 0,072 & 0,340 & 0,258 & 0,127 & 0,361 \\
\hline Jornada completa & 0,722 & 0,928 & 0,660 & 0,742 & 0,873 & 0,639 \\
\hline Supervisión & 0,136 & 0,081 & 0,061 & 0,152 & 0,116 & 0,088 \\
\hline No supervisión & 0,864 & 0,919 & 0,939 & 0,848 & 0,884 & 0,912 \\
\hline Directores y gerentes & 0,014 & 0,009 & 0,004 & 0,023 & 0,023 & 0,013 \\
\hline $\begin{array}{l}\text { Técnicos y profesionales } \\
\text { científicos }\end{array}$ & 0,170 & 0,029 & 0,061 & 0,192 & 0,085 & 0,108 \\
\hline $\begin{array}{l}\text { Técnicos y profesionales } \\
\text { de apoyo }\end{array}$ & 0,147 & 0,041 & 0,080 & 0,175 & 0,091 & 0,103 \\
\hline $\begin{array}{l}\text { Empleados } \\
\text { administrativos } \\
\text { y de oficina }\end{array}$ & 0,195 & 0,028 & 0,112 & 0,200 & 0,052 & 0,154 \\
\hline $\begin{array}{l}\text { Servicios de restauración } \\
\text { y vendedores }\end{array}$ & 0,193 & 0,075 & 0,268 & 0,197 & 0,110 & 0,258 \\
\hline $\begin{array}{l}\text { Trabajadores cualificados } \\
\text { en agricultura }\end{array}$ & 0,000 & 0,003 & 0,001 & 0,001 & 0,007 & 0,001 \\
\hline $\begin{array}{l}\text { Trabajadores cualificados } \\
\text { de manufacturas y } \\
\text { construcción }\end{array}$ & 0,044 & 0,343 & 0,066 & 0,034 & 0,274 & 0,044 \\
\hline $\begin{array}{l}\text { Operadores de } \\
\text { instalaciones y } \\
\text { maquinaria }\end{array}$ & 0,059 & 0,167 & 0,059 & 0,052 & 0,128 & 0,043 \\
\hline $\begin{array}{l}\text { Ocupaciones } \\
\text { elementales/No } \\
\text { cualificados } \\
\end{array}$ & 0,178 & 0,305 & 0,349 & 0,125 & 0,230 & 0,276 \\
\hline Andalucía & 0,088 & 0,039 & 0,046 & 0,101 & 0,043 & 0,051 \\
\hline Aragón & 0,041 & 0,074 & 0,053 & 0,035 & 0,050 & 0,038 \\
\hline Asturias & 0,027 & 0,010 & 0,008 & 0,022 & 0,014 & 0,008 \\
\hline Baleares & 0,028 & 0,056 & 0,047 & 0,027 & 0,051 & 0,057 \\
\hline
\end{tabular}

NOTA: Entre paréntesis aparece la desviación estándar de las variables continuas. 
CUADRO A.1 (continuación)

DESCRIPTIVOS DE LAS VARIABLES

\begin{tabular}{|c|c|c|c|c|c|c|}
\hline & \multicolumn{3}{|c|}{2006} & \multicolumn{3}{|c|}{2010} \\
\hline & $\begin{array}{l}\text { Mujer } \\
\text { nativa }\end{array}$ & $\begin{array}{l}\text { Hombre } \\
\text { inmigrante }\end{array}$ & $\begin{array}{c}\text { Mujer } \\
\text { inmigrante }\end{array}$ & $\begin{array}{l}\text { Mujer } \\
\text { nativa }\end{array}$ & $\begin{array}{l}\text { Hombre } \\
\text { inmigrante }\end{array}$ & $\begin{array}{c}\text { Mujer } \\
\text { inmigrante }\end{array}$ \\
\hline Canarias & 0,042 & 0,042 & 0,062 & 0,038 & 0,043 & 0,057 \\
\hline Cantabria & 0,020 & 0,017 & 0,015 & 0,017 & 0,013 & 0,008 \\
\hline Castilla-La Mancha & 0,043 & 0,058 & 0,035 & 0,035 & 0,038 & 0,023 \\
\hline Castilla y León & 0,060 & 0,042 & 0,025 & 0,049 & 0,036 & 0,022 \\
\hline Cataluña & 0,169 & 0,175 & 0,200 & 0,199 & 0,240 & 0,259 \\
\hline Comunidad Valenciana & 0,088 & 0,098 & 0,106 & 0,081 & 0,071 & 0,079 \\
\hline Extremadura & 0,022 & 0,009 & 0,005 & 0,018 & 0,011 & 0,005 \\
\hline Galicia & 0,060 & 0,023 & 0,015 & 0,055 & 0,022 & 0,014 \\
\hline Madrid & 0,166 & 0,182 & 0,239 & 0,209 & 0,239 & 0,298 \\
\hline Murcia & 0,039 & 0,073 & 0,054 & 0,026 & 0,028 & 0,022 \\
\hline Navarra & 0,024 & 0,040 & 0,039 & 0,022 & 0,031 & 0,019 \\
\hline País Vasco & 0,066 & 0,026 & 0,028 & 0,051 & 0,033 & 0,019 \\
\hline La Rioja & 0,016 & 0,033 & 0,022 & 0,013 & 0,027 & 0,018 \\
\hline Ceuta y Melilla & 0,001 & 0,004 & 0,001 & 0,002 & 0,010 & 0,003 \\
\hline Industrias extractivas & 0,002 & 0,010 & 0,002 & 0,001 & 0,007 & 0,001 \\
\hline Industria manufacturera & 0,198 & 0,288 & 0,160 & 0,195 & 0,269 & 0,145 \\
\hline $\begin{array}{l}\text { Producción de energía } \\
\text { eléctrica, gas y agua }\end{array}$ & 0,003 & 0,001 & 0,000 & 0,012 & 0,020 & 0,007 \\
\hline Construcción & 0,022 & 0,349 & 0,015 & 0,021 & 0,207 & 0,014 \\
\hline Comercio & 0,116 & 0,051 & 0,101 & 0,147 & 0,123 & 0,144 \\
\hline Hostelería & 0,084 & 0,103 & 0,294 & 0,040 & 0,065 & 0,126 \\
\hline $\begin{array}{l}\text { Transporte y } \\
\text { comunicaciones }\end{array}$ & 0,025 & 0,068 & 0,020 & 0,054 & 0,035 & 0,041 \\
\hline $\begin{array}{l}\text { Intermediación } \\
\text { financiera }\end{array}$ & 0,051 & 0,005 & 0,014 & 0,050 & 0,007 & 0,016 \\
\hline $\begin{array}{l}\text { Actividades } \\
\text { inmobiliarias y de } \\
\text { alquiler }\end{array}$ & 0,183 & 0,089 & 0,219 & 0,232 & 0,188 & 0,313 \\
\hline Educación & 0,103 & 0,016 & 0,050 & 0,032 & 0,009 & 0,019 \\
\hline Sanidad & 0,175 & 0,007 & 0,090 & 0,163 & 0,020 & 0,106 \\
\hline $\begin{array}{l}\text { Otras actividades } \\
\text { sociales y de servicios }\end{array}$ & 0,037 & 0,015 & 0,035 & 0,054 & 0,050 & 0,067 \\
\hline Tamaño menos de 20 & 0,183 & 0,296 & 0,235 & 0,208 & 0,297 & 0,244 \\
\hline Tamaño 20-49 & 0,191 & 0,299 & 0,236 & 0,100 & 0,170 & 0,112 \\
\hline Tamaño 50-99 & 0,120 & 0,150 & 0,126 & 0,083 & 0,127 & 0,092 \\
\hline Tamaño 100-199 & 0,101 & 0,107 & 0,114 & 0,112 & 0,123 & 0,115 \\
\hline Tamaño 200-499 & 0,167 & 0,075 & 0,117 & 0,217 & 0,165 & 0,209 \\
\hline Tamaño 500 ó más & 0,238 & 0,072 & 0,172 & 0,279 & 0,118 & 0,228 \\
\hline $\begin{array}{l}\text { Convenio sectorial de } \\
\text { ámbito nacional }\end{array}$ & 0,427 & 0,345 & 0,380 & 0,431 & 0,368 & 0,432 \\
\hline $\begin{array}{l}\text { Convenio sectorial de } \\
\text { ámbito infranacional }\end{array}$ & 0,499 & 0,639 & 0,581 & 0,454 & 0,559 & 0,460 \\
\hline Convenio de empresa & 0,074 & 0,016 & 0,039 & 0,115 & 0,072 & 0,108 \\
\hline $\begin{array}{l}\text { Número de } \\
\text { observaciones }\end{array}$ & 53.409 & 6.876 & 3.417 & 63.038 & 6.584 & 4.272 \\
\hline
\end{tabular}

NOTA: Entre paréntesis aparece la desviación está ndar de las variables continuas. 
CUADRO A.2

DESCOMPOSICIÓN DE LAS DIFERENCIAS SALARIALES

MUJERES NATIVAS E INMIGRANTES

(Modelo 1.2006)

\begin{tabular}{|l|l|c|c|c|}
\hline \multicolumn{2}{|c|}{} & \multicolumn{3}{c|}{ Cuantiles } \\
\cline { 3 - 5 } \multicolumn{2}{|c|}{ Total } & Porcentil 10 & Mediana & Percentil 90 \\
\cline { 2 - 5 } & Mujeres inmigrantes & $\begin{array}{c}1,517 \\
(0,002)^{* * *}\end{array}$ & $\begin{array}{c}1,966 \\
(0,003)^{* * *}\end{array}$ & $\begin{array}{c}2,957 \\
(0,005)^{* * *}\end{array}$ \\
\cline { 2 - 5 } & Diferencia & $\begin{array}{c}1,478 \\
(0,007)^{* * *}\end{array}$ & $\begin{array}{c}1,790 \\
(0,006)^{* * *}\end{array}$ & $\begin{array}{c}2,633 \\
(0,028)^{* * *}\end{array}$ \\
\cline { 2 - 5 } & Características & $\begin{array}{c}0,039 \\
(0,007)^{* * *}\end{array}$ & $\begin{array}{c}0,176 \\
(0,007)^{* * *}\end{array}$ & $\begin{array}{c}0,325 \\
(0,029)^{* * *}\end{array}$ \\
\cline { 2 - 5 } & Coeficientes & $\begin{array}{c}0,050 \\
(0,002)^{* * *}\end{array}$ & $\begin{array}{c}0,141 \\
(0,005)^{* * *}\end{array}$ & $\begin{array}{c}0,154 \\
(0,006)^{* * *}\end{array}$ \\
\cline { 2 - 5 } & Edad & $\begin{array}{c}-0,011 \\
(0,007)\end{array}$ & $\begin{array}{c}0,035 \\
(0,007)^{* * *}\end{array}$ & $\begin{array}{c}0,170 \\
(0,028)^{* * *}\end{array}$ \\
\cline { 2 - 5 } & Educación & $\begin{array}{c}0,009 \\
(0,001)^{* * *}\end{array}$ & $\begin{array}{c}0,026 \\
(0,002)^{* * *}\end{array}$ & $\begin{array}{c}0,039 \\
(0,003)^{* * *}\end{array}$ \\
\cline { 2 - 5 } & & $\begin{array}{c}0,040 \\
(0,002)^{* * *}\end{array}$ & $\begin{array}{c}0,115 \\
(0,004)^{* * *}\end{array}$ & $\begin{array}{c}0,115 \\
(0,005)^{* * *}\end{array}$ \\
\cline { 2 - 5 } & & 56,826 & 56,826 & 56,826 \\
\hline
\end{tabular}

NOTAS: $* p<0,1 ; * * p<0,05 ; * * * p<0,01$ 
CUADRO A.3

DESCOMPOSICIÓN DE LAS DIFERENCIAS SALARIALES

MUJERES NATIVAS E INMIGRANTES

(Modelo 1.2010)

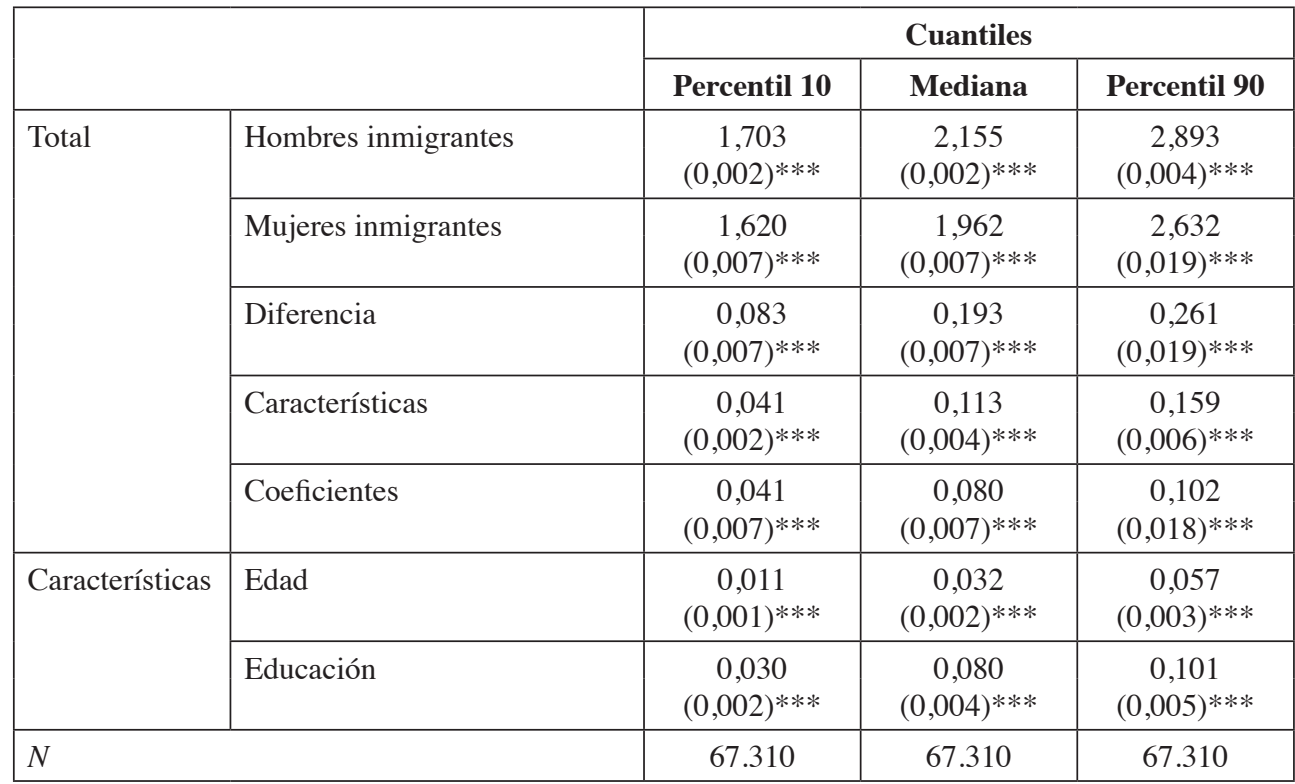

NOTAS: $* p<0,1 ; * * p<0,05 ; * * * p<0,01$. 
CUADRO A.4

DESCOMPOSICIÓN DE LAS DIFERENCIAS SALARIALES HOMBRES Y MUJERES INMIGRANTES

(Modelo 1.2006)

\begin{tabular}{|l|l|c|c|c|}
\hline \multicolumn{2}{|c|}{} & \multicolumn{3}{c|}{ Cuantiles } \\
\cline { 3 - 5 } \multicolumn{2}{|c|}{ Total } & Percentil 10 & Mediana & Percentil 90 \\
\cline { 2 - 5 } & Hombres inmigrantes & $\begin{array}{c}1,635 \\
(0,005)^{* * *}\end{array}$ & $\begin{array}{c}1,923 \\
(0,004)^{* * *}\end{array}$ & $\begin{array}{c}2,460 \\
(0,014)^{* * *}\end{array}$ \\
\cline { 2 - 5 } & Mujeres inmigrantes & $\begin{array}{c}1,478 \\
(0,007)^{* * *}\end{array}$ & $\begin{array}{c}1,790 \\
(0,006)^{* * *}\end{array}$ & $\begin{array}{c}2,382 \\
(0,021)^{* * *}\end{array}$ \\
\cline { 2 - 5 } & Diferencia & $\begin{array}{c}0,157 \\
(0,008)^{* * *}\end{array}$ & $\begin{array}{c}0,133 \\
(0,007)^{* * *}\end{array}$ & $\begin{array}{c}0,077 \\
(0,025)^{* * *}\end{array}$ \\
\cline { 2 - 5 } & Características & $\begin{array}{c}-0,005 \\
(0,001)^{* * *}\end{array}$ & $\begin{array}{c}-0,014 \\
(0,002)^{* * *}\end{array}$ & $\begin{array}{c}-0,087 \\
(0,011)^{* * *}\end{array}$ \\
\cline { 2 - 5 } & Coeficientes & $\begin{array}{c}0,162 \\
(0,008)^{* * *}\end{array}$ & $\begin{array}{c}0,147 \\
(0,007)^{* * *}\end{array}$ & $\begin{array}{c}0,164 \\
(0,023)^{* * * *}\end{array}$ \\
\cline { 2 - 5 } & Earacterísticas & $\begin{array}{c}0,002 \\
(0,001)^{* * *}\end{array}$ & $\begin{array}{c}0,002 \\
(0,001)^{* * *}\end{array}$ & $\begin{array}{c}0,008 \\
(0,003)^{* * * *}\end{array}$ \\
\cline { 2 - 5 } & Edad & $\begin{array}{c}-0,006 \\
(0,001)^{* * *}\end{array}$ & $\begin{array}{c}-0,016 \\
(0,002)^{* * *}\end{array}$ & $\begin{array}{c}-0,095 \\
(0,010)^{* * *}\end{array}$ \\
\cline { 2 - 5 } & Educación & 10.293 & 10.293 & 10.293 \\
\hline
\end{tabular}

NOTAS: $* p<0,1 ; * * p<0,05 ; * * * p<0,01$ 
CUADRO A.5

DESCOMPOSICIÓN DE LAS DIFERENCIAS SALARIALES HOMBRES Y MUJERES INMIGRANTES

(Modelo 1.2010)

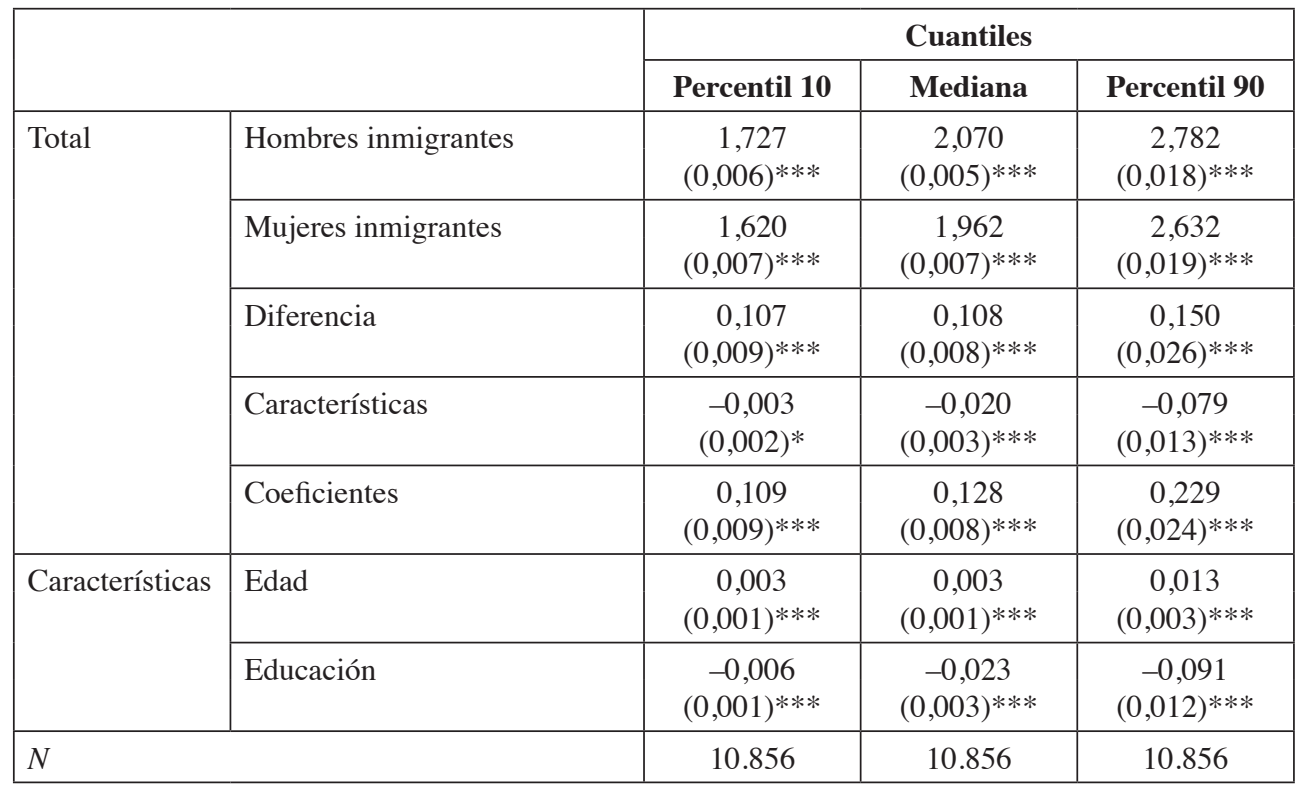

NOTAS: $* p<0,1 ; * * p<0,05 ; * * * p<0,01$. 
CUADRO A.6

DESCOMPOSICIÓN DE LAS DIFERENCIAS SALARIALES HOMBRES Y MUJERES INMIGRANTES

(Modelo 2. 2006)

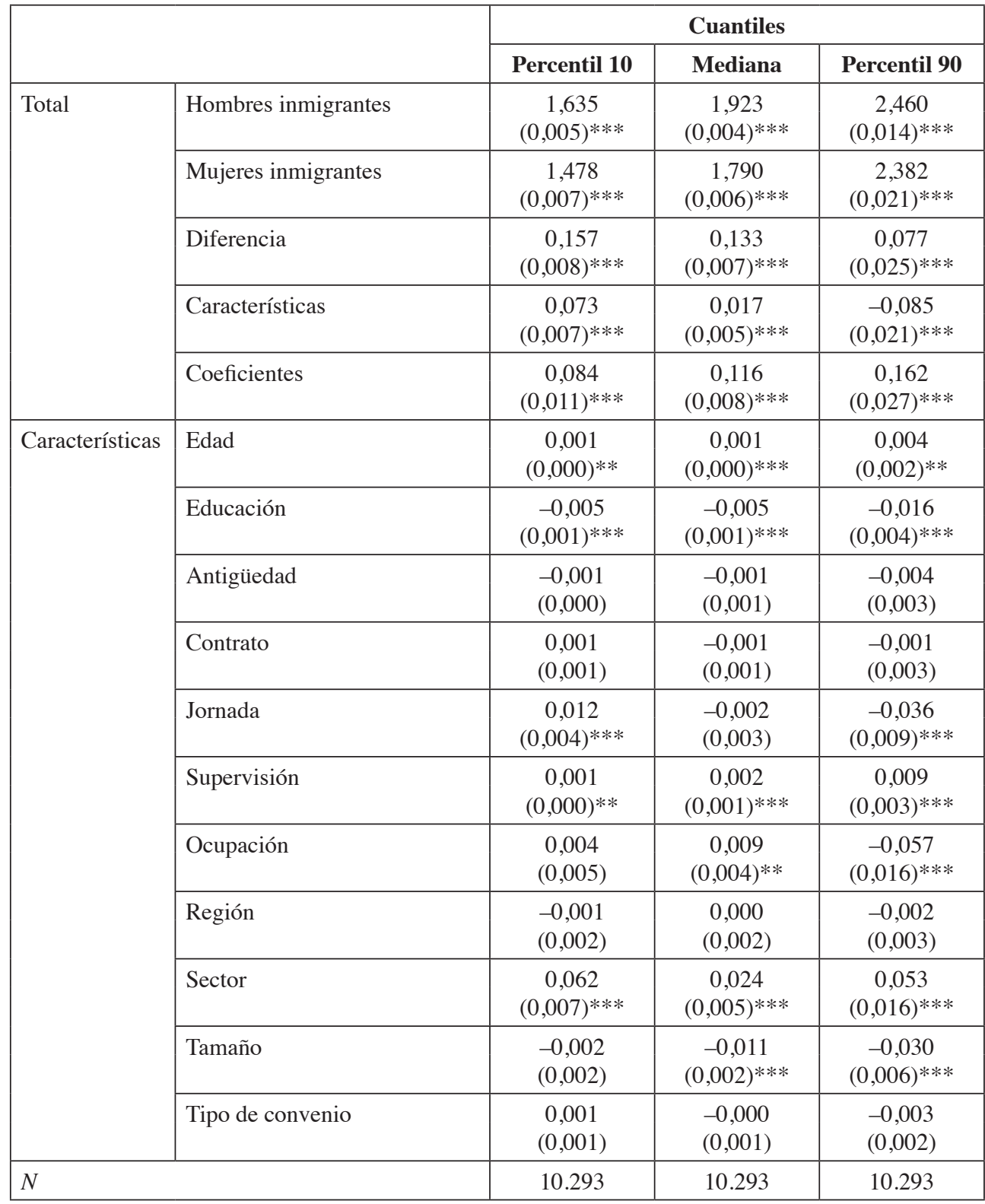

NOTAS: $* p<0,1 ; * * p<0,05 ; * * * p<0,01$ 
CUADRO A.7

DESCOMPOSICIÓN DE LAS DIFERENCIAS SALARIALES HOMBRES Y MUJERES INMIGRANTES

(Modelo 2. 2010)

\begin{tabular}{|c|c|c|c|c|}
\hline & \multirow[b]{2}{*}{ Percentil 10} & \multicolumn{2}{|l|}{ Cuantiles } \\
\hline & & & Mediana & Percentil 90 \\
\hline \multirow[t]{5}{*}{ Total } & Hombres inmigrantes & $\begin{array}{c}1,727 \\
(0,006) * * *\end{array}$ & $\begin{array}{c}2,070 \\
(0,005)^{* * *}\end{array}$ & $\begin{array}{c}2,782 \\
(0,018) * * *\end{array}$ \\
\hline & Mujeres inmigrantes & $\begin{array}{c}1,620 \\
(0,007) * * *\end{array}$ & $\begin{array}{c}1,962 \\
(0,007)^{* * *}\end{array}$ & $\begin{array}{c}2,632 \\
(0,019)^{* * *}\end{array}$ \\
\hline & Diferencia & $\begin{array}{c}0,107 \\
(0,009) * * *\end{array}$ & $\begin{array}{c}0,108 \\
(0,008)^{* * *}\end{array}$ & $\begin{array}{c}0,150 \\
(0,026) * * *\end{array}$ \\
\hline & Características & $\begin{array}{c}0,060 \\
(0,006) * * *\end{array}$ & $\begin{array}{c}0,026 \\
(0,006) * * *\end{array}$ & $\begin{array}{c}-0,033 \\
(0,020)^{*}\end{array}$ \\
\hline & Coeficientes & $\begin{array}{c}0,046 \\
(0,011)^{* * *}\end{array}$ & $\begin{array}{c}0,082 \\
(0,008) * * *\end{array}$ & $\begin{array}{c}0,183 \\
(0,026)^{* * *}\end{array}$ \\
\hline \multirow[t]{11}{*}{ Características } & Edad & $\begin{array}{c}0,002 \\
(0,001)^{* * *}\end{array}$ & $\begin{array}{c}0,001 \\
(0,000)^{* *}\end{array}$ & $\begin{array}{c}0,006 \\
(0,002)^{* * *}\end{array}$ \\
\hline & Educación & $\begin{array}{c}-0,003 \\
(0,001)^{* *}\end{array}$ & $\begin{array}{c}-0,007 \\
(0,001)^{* * *}\end{array}$ & $\begin{array}{c}-0,025 \\
(0,005)^{* * *}\end{array}$ \\
\hline & Antigüedad & $\begin{array}{l}-0,001 \\
(0,001)\end{array}$ & $\begin{array}{l}-0,001 \\
(0,001)\end{array}$ & $\begin{array}{l}-0,002 \\
(0,004)\end{array}$ \\
\hline & Contrato & $\begin{array}{c}0,000 \\
(0,001)\end{array}$ & $\begin{array}{c}0,001 \\
(0,001)\end{array}$ & $\begin{array}{c}0,000 \\
(0,002)\end{array}$ \\
\hline & Jornada & $\begin{array}{c}0,005 \\
(0,003)\end{array}$ & $\begin{array}{c}0,004 \\
(0,002)\end{array}$ & $\begin{array}{c}-0,030 \\
(0,006)^{* * *}\end{array}$ \\
\hline & Supervisión & $\begin{array}{c}0,001 \\
(0,000)^{* * *}\end{array}$ & $\begin{array}{c}0,002 \\
(0,001)^{* * *}\end{array}$ & $\begin{array}{c}0,007 \\
(0,002)^{* * *}\end{array}$ \\
\hline & Ocupación & $\begin{array}{c}0,024 \\
(0,005)^{* * *}\end{array}$ & $\begin{array}{c}0,015 \\
(0,004)^{* * *}\end{array}$ & $\begin{array}{l}-0,024 \\
(0,015)\end{array}$ \\
\hline & Región & $\begin{array}{c}0,003 \\
(0,002)^{*}\end{array}$ & $\begin{array}{c}0,003 \\
(0,002)^{*}\end{array}$ & $\begin{array}{r}0,004 \\
(0,003) \\
\end{array}$ \\
\hline & Sector & $\begin{array}{c}0,037 \\
(0,004) * * *\end{array}$ & $\begin{array}{c}0,019 \\
(0,004) * * *\end{array}$ & $\begin{array}{c}0,053 \\
(0,012)^{* * *}\end{array}$ \\
\hline & Tamaño & $\begin{array}{c}-0,017 \\
(0,002) * * *\end{array}$ & $\begin{array}{c}-0,012 \\
(0,002) * * * \\
\end{array}$ & $\begin{array}{c}-0,020 \\
(0,005) * * * \\
\end{array}$ \\
\hline & Tipo de convenio & $\begin{array}{c}0,009 \\
(0,002) * * *\end{array}$ & $\begin{array}{c}0,001 \\
(0,001)\end{array}$ & $\begin{array}{l}-0,002 \\
(0,003)\end{array}$ \\
\hline \multicolumn{2}{|l|}{$N$} & 10.856 & 10.856 & 10.856 \\
\hline
\end{tabular}

NOTAS: $* p<0,1 ; * * p<0,05 ; * * * p<0,01$ 
CUADRO A.8

DESCOMPOSICIÓN DE LAS DIFERENCIAS SALARIALES MUJERES NATIVAS E INMIGRANTES

(Modelo 2. 2006)

\begin{tabular}{|c|c|c|c|c|}
\hline & \multicolumn{3}{|c|}{ Cuantiles } \\
\hline & & Percentil 10 & Mediana & Percentil 90 \\
\hline \multirow[t]{5}{*}{ Total } & Mujeres nativas & $\begin{array}{c}1,517 \\
(0,002)^{* * *}\end{array}$ & $\begin{array}{c}1,966 \\
(0,003)^{* * *}\end{array}$ & $\begin{array}{c}2,758 \\
(0,004)^{* * *}\end{array}$ \\
\hline & Mujeres inmigrantes & $\begin{array}{c}1,478 \\
(0,007)^{* * *}\end{array}$ & $\begin{array}{c}1,790 \\
(0,006) * * *\end{array}$ & $\begin{array}{c}2,382 \\
(0,021)^{* * *}\end{array}$ \\
\hline & Diferencia & $\begin{array}{c}0,039 \\
(0,007)^{* * *}\end{array}$ & $\begin{array}{c}0,176 \\
(0,007)^{* * *}\end{array}$ & $\begin{array}{c}0,376 \\
(0,022)^{* * *}\end{array}$ \\
\hline & Características & $\begin{array}{c}0,068 \\
(0,003)^{* * *}\end{array}$ & $\begin{array}{c}0,250 \\
(0,006) * * *\end{array}$ & $\begin{array}{c}0,282 \\
(0,008)^{* * *}\end{array}$ \\
\hline & Coeficientes & $\begin{array}{c}-0,029 \\
(0,008) * * *\end{array}$ & $\begin{array}{c}-0,074 \\
(0,007) * * *\end{array}$ & $\begin{array}{c}0,094 \\
(0,020)^{* * *}\end{array}$ \\
\hline \multirow[t]{11}{*}{ Características } & Edad & $\begin{array}{c}0,003 \\
(0,001)^{* * *}\end{array}$ & $\begin{array}{c}0,005 \\
(0,001)^{* * *}\end{array}$ & $\begin{array}{c}0,018 \\
(0,002)^{* * *}\end{array}$ \\
\hline & Educación & $\begin{array}{c}0,020 \\
(0,002) * * *\end{array}$ & $\begin{array}{c}0,032 \\
(0,002) * * *\end{array}$ & $\begin{array}{c}0,029 \\
(0,002) * * *\end{array}$ \\
\hline & Antigüedad & $\begin{array}{c}0,036 \\
(0,002)^{* * *}\end{array}$ & $\begin{array}{c}0,087 \\
(0,002) * * *\end{array}$ & $\begin{array}{c}0,084 \\
(0,004)^{* * *}\end{array}$ \\
\hline & Contrato & $\begin{array}{c}0,000 \\
(0,001)\end{array}$ & $\begin{array}{c}0,000 \\
(0,001)\end{array}$ & $\begin{array}{c}0,001 \\
(0,002)\end{array}$ \\
\hline & Jornada & $\begin{array}{c}0,005 \\
(0,001)^{* * *}\end{array}$ & $\begin{array}{c}0,003 \\
(0,000) * * *\end{array}$ & $\begin{array}{c}-0,004 \\
(0,001)^{* * *}\end{array}$ \\
\hline & Supervisión & $\begin{array}{c}0,002 \\
(0,000)^{* * *}\end{array}$ & $\begin{array}{c}0,010 \\
(0,001)^{* * *}\end{array}$ & $\begin{array}{c}0,008 \\
(0,001)^{* * *}\end{array}$ \\
\hline & Ocupación & $\begin{array}{c}0,027 \\
(0,002)^{* * *}\end{array}$ & $\begin{array}{c}0,091 \\
(0,003)^{* * *}\end{array}$ & $\begin{array}{c}0,118 \\
(0,005) * * *\end{array}$ \\
\hline & Región & $\begin{array}{c}-0,008 \\
(0,001)^{* * *} \\
\end{array}$ & $\begin{array}{c}-0,008 \\
(0,001)^{* * *}\end{array}$ & $\begin{array}{c}-0,004 \\
(0,002)^{* *}\end{array}$ \\
\hline & Sector & $\begin{array}{c}-0,024 \\
(0,002)^{* * *}\end{array}$ & $\begin{array}{c}0,008 \\
(0,002) * * *\end{array}$ & $\begin{array}{c}0,022 \\
(0,003)^{* * *}\end{array}$ \\
\hline & Tamaño & $\begin{array}{c}0,006 \\
(0,001)^{* * *} \\
\end{array}$ & $\begin{array}{c}0,021 \\
(0,002)^{* * *}\end{array}$ & $\begin{array}{c}0,007 \\
(0,001)^{* * *}\end{array}$ \\
\hline & Tipo de convenio & $\begin{array}{c}0,001 \\
(0,000) \\
\end{array}$ & $\begin{array}{c}0,003 \\
(0,001) * * * \\
\end{array}$ & $\begin{array}{c}0,003 \\
(0,001)^{* * *}\end{array}$ \\
\hline \multicolumn{2}{|l|}{$N$} & 56.826 & 56.826 & 56.826 \\
\hline
\end{tabular}

NOTAS: $* p<0,1 ; * * p<0,05 ; * * * p<0,01$ 
CUADRO A.9

DESCOMPOSICIÓN DE LAS DIFERENCIAS SALARIALES MUJERES NATIVAS E INMIGRANTES

(Modelo 2. 2010)

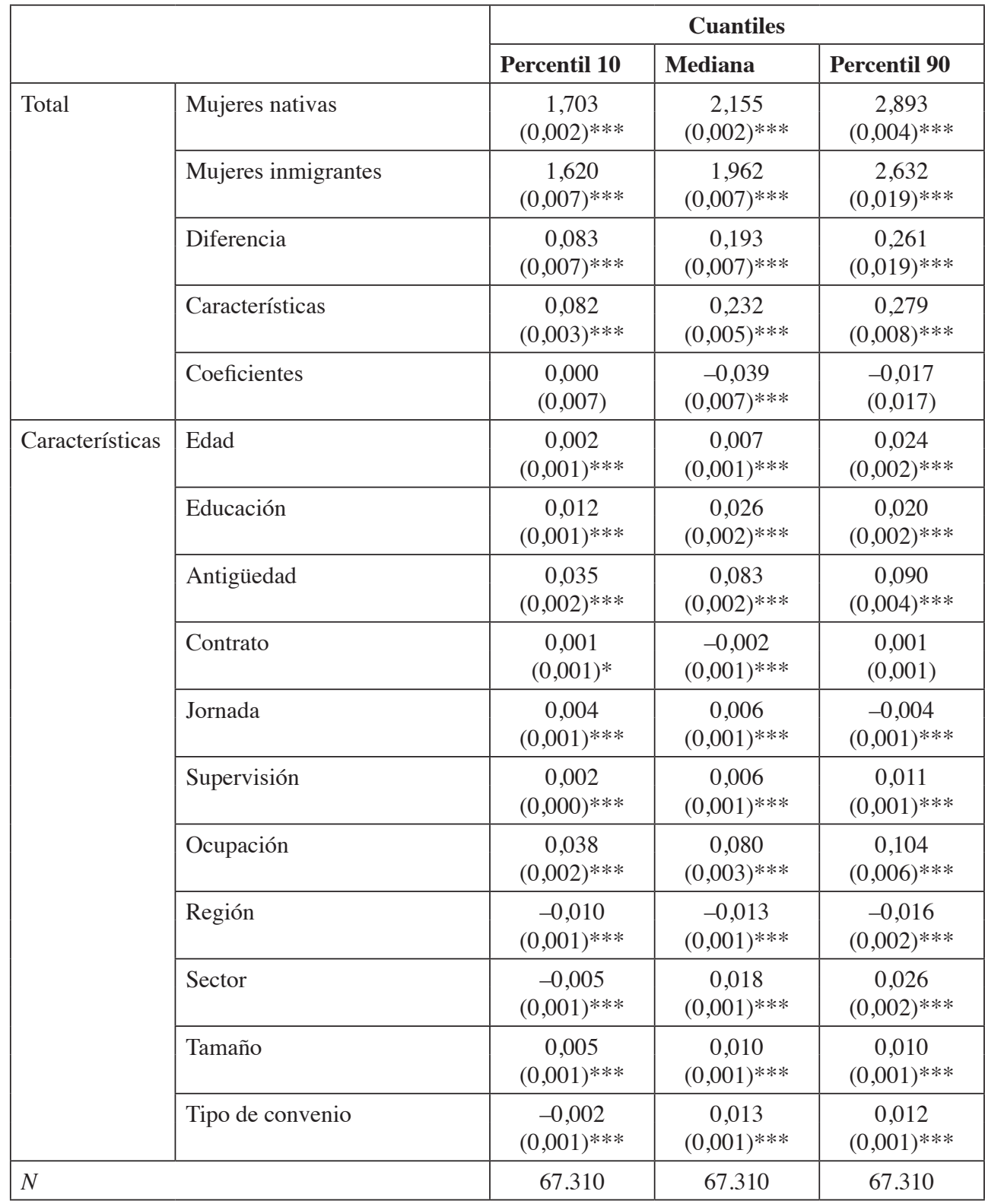

NOTAS: $* p<0,1 ; * * p<0,05 ; * * * p<0,01$. 